\author{
Series A
}

I. MATHEMATICA

401

\title{
DAS SPEKTRUM ZWEIER GEKOPPELTER ZUEINANDER ADJUNGIERTER ELLIPTISCHER DIFFERENTIALGLEICHUNGEN ZWEITER ORDNUNG
}

LORENTZ-SOMMERFELDSCHE VERMUTUNG

VON

ERNST MOHR 
Am 12. November 1965 vorgelegt von P. J. MYrberg und K. VëIsäÏ̈ 


\section{Stellung der Aufgabe, Bezeichnungen und Lösungsmethode}

1. In einem beschränkten Gebiet der $x y$-Ebene (wir beschränken uns auf den Fall der Ebene) seien in der üblichen Schreibung $(u=u(x, y)$, $\left.u_{x}=\partial u / \partial x, \ldots, \Delta u=u_{x x}+u_{y y}\right)$ die zueinander adjungierten Differentialausdrücke

$$
\begin{aligned}
L[u] & =\Delta u+a u_{x}+b u_{y}+c u \\
M[v] & =\Delta v-(a v)_{x}-(b v)_{y}+c v \\
& =\Delta v-a v_{x}-b v_{y}+\left(c-a_{x}-b_{y}\right) v
\end{aligned}
$$

gegeben; $\partial / \partial n$ bedeute die Ableitung nach der äusseren Normalen $\{\alpha, \beta\}$. Dann handelt es sich um das Paar von Differentialgleichungen

$$
\begin{aligned}
& L[u]+\lambda v=0, \\
& M[v]+\lambda u=0,
\end{aligned}
$$

wobei $\lambda$ ein Parameter ist; für $u$ wird eine Randbedingung

$$
\frac{\partial u}{\partial n}+\sigma u=0
$$

gestellt; für $v$ ergibt sich daraus die adjungierte Randbedingung

$$
\frac{\partial v}{\partial n}+\tau v=0 \quad \text { mit } \quad \tau=\sigma-(a \alpha+b \beta) .
$$

Gefragt wird nach allen Eigenwerten $\lambda$ mit zugehörigen Eigenfunktionen $u, v$, für welche die beiden Differentialgleichungen und die Randbedingungen erfüllt sind. Da mit $\lambda$ und $u, v$ immer auch $-\lambda$ und $u,-v$ Lösungen sind, darf $\lambda \geqq 0$ angenommen werden. Die Randbedingungen (1a) und ( $1 \mathrm{~b})$ sind natürlich im Courantschen Sinne zu verstehen: man vergleiche dazu Courant-Hilbert [2], Seite 508 ff. Die Randintegrale, die im folgenden auftreten, können nach Courant durch ein Gebietsintegral über einen Divergenzausdruck ersetzt werden, was für die Existenzbeweise wichtig ist; hier jedoch können und wollen wir die Randintegrale mitführen.

Für den Fall, dass die Randbedingungen durch $u=0, v=0$ gegeben sind, haben wir in einer früheren Arbeit [3] das asymptotische Verteilungs- 
gesetz, das heisst die Lorentz-Sommerfeldsche Vermutung, für die Eigenwerte $\lambda_{p}(p=1,2, \ldots)$ bewiesen:

$$
\lambda_{p} \cong \frac{4 \pi p}{F} \quad \text { d. h. } \quad \lim \frac{\lambda_{p} F}{4 \pi p}=1
$$

für $p \rightarrow \infty$, wobei $F$ der Jordansche Inhalt des Gebietes ist. Dieser Beweis setzte die Existenz von zugehörigen Eigenfunktionen nicht voraus, und arbeitete nur mit dem Begriff der Eigenfolge anstelle von Eigenfunktion. Wir zeigen im folgenden, dass wir auf der Linie dieser Überlegungen auch für die jetzt vorliegenden Randbedingungen (1a) und (1b) das asymptotische Gesetz (2) begründen können. Dabei treten gegenüber der früheren Arbeit [3] neue Schwierigkeiten auf, die dadurch bedingt sind, dass ständig gewisse Randintegrale auftreten, die besonders behandelt und abgeschätzt werden müssen.

2. In den Bezeichnungen und in der Schreibung von Integralen (Unterdrückung des jeweiligen Integrationselementes) schliessen wir uns weitgehend an die erste Arbeit an und übernehmen deren Ergebnisse, soweit dies möglich ist. $O(1)$ und $o(1)$ sind die bekannten Landauschen Symbole. Ein flächenhaftes Integral wird stets durch zwei, ein linienhaftes stets durch ein Integralzeichen angedeutet. So schreiben wir z. B. die integrale Bilinearform, welche zu unserer Aufgabe gehört, wie folgt:

$$
I(u ; v)=\iint\left\{u_{x} v_{x}+u_{y} v_{y}-a v u_{x}-b v u_{y}+c v u\right\}+\int \sigma v u ;
$$

und analog die bekannten Integrale

$$
\begin{aligned}
D[u, v] & =\iint\left(u_{x} v_{x}+u_{y} v_{y}\right), & D[u, u]=D[u], \\
H[u, v] & =\iint u v, & H[u, u]=H[u], \\
H[u, u] & =\|u\|^{2} . &
\end{aligned}
$$

Besitzt eine Integralform wie $I(u ; v)$ ein Randintegral, so bezeichnen wir den flächenhaften Beitrag durch $I^{*}(u ; v)$, schreiben also

$$
I^{*}(u ; v)=\iint\left\{u_{x} v_{x}+u_{y} v_{y}-a v u_{x}-b v u_{y}+c v u\right\} .
$$

Mit $\Omega$ bezeichnen wir den in Courant-Hilbert [2], S. 479, definierten Raum

$$
\Omega=\{f \mid H[f]<\infty, D[f]<\infty\} .
$$


Handelt es sich bei einer bestimmten Aufgabe um eine Familie von Funktionen $f$ (im allgemeinen wird diese Familie durch eine Folge $f^{n}$ gegeben sein), deren $H$ - und $D$-Integrale gleichgradig beschränkt sind, so bezeichnen wir den betreffenden Raum durch $E$ :

$$
E=\left\{f \mid H[f] \leqq \Gamma_{1}, D[f] \leqq \Gamma_{2}\right\}
$$

$\Gamma_{1}>0$ und $\Gamma_{2}>0$ bezeichnen bei der betreffenden Aufgabe absolute Konstante, die also von dem einzelnen Element $f$ nicht abhängen. Liegt speziell eine Funktionenfolge $f^{n}$ vor, so drücken wir die gleichgradige Beschränktheit von $H\left[f^{n}\right]$ und $D\left[f^{n}\right]$ auch durch

$$
H\left[f^{n}\right]=O(1), \quad D\left[f^{n}\right]=O(1)
$$

aus. Ist eine zweite Folge $g^{n}$ mit den gleichen Eigenschaften gegeben

$$
H\left[g^{n}\right]=O(1), \quad D\left[g^{n}\right]=O(1),
$$

so drücken wir für die Folge von Funktionenpaaren $\left(f^{n}, g^{n}\right)$ diesen Tatbestand kurz durch

$$
\left(f^{n}, g^{n}\right) \in E
$$

aus.

Gilt speziell für die Folge $f^{n}$

$$
H\left[f^{n}\right]=o(1), \quad D\left[f^{n}\right]=o(1),
$$

so sagen wir: $f^{n}$ ist äquivalent $N u l l$, in Zeichen

$$
f^{n} \sim 0
$$

ähnlich schreiben wir für zwei Folgen $f^{n}$ und $g^{n}$

$$
f^{n} \sim g^{n} \text { falls } f^{n}-g^{n} \sim 0 .
$$

Man sieht sofort, dass es sich um einen Äquivalenzbegriff handelt. Da im folgenden häufig Paare von Funktionen bzw. Funktionenfolgen $\left(u^{n}, v^{n}\right)$ auftreten, so schreiben wir auch

$$
\left(u^{n}, v^{n}\right) \sim(0,0) \quad \text { für } \quad u^{n} \sim 0, v^{n} \sim 0 .
$$

3. Das Gleichungspaar (1), zusammen mit den Randbedingungen (1a) und (1b) ergibt sich als ein Euler-Lagrangesches Paar von Aussagen, das $\mathrm{zu}$ dem folgenden freien Variationsproblem gehört: gesucht sind alle Funktionenpaare $(u, v)$ mit $\|u\|^{2}+\|v\|^{2}=2$, für welche die Bilinearform $I(u ; v)$ in $(3)$ stationär wird:

$$
I(u ; v)=\text { stationär bei }\|u\|^{2}+\|v\|^{2}=2 .
$$


In der Tat ergeben sich mit $\lambda / 2$ als Multiplikator aus der Forderung

$$
I(u ; v)-\frac{\lambda}{2}\left\{\iint u^{2}+\iint v^{2}\right\}=\text { stationär }
$$

die noch nicht ausintegrierten »Variationsgleichungen»

$$
\left\{\begin{array}{l}
I(\zeta ; v)-\lambda \iint \zeta u=0, \\
I(u ; \eta)-\lambda \iint v \eta=0,
\end{array}\right.
$$

wobei $\zeta, \eta$ beliebige Elemente aus $\Omega$ sind. Wegen

$$
\begin{aligned}
& I(\zeta ; v)=\iint(-\zeta M[v])+\int \zeta\left\{\frac{\partial v}{\partial n}+\tau v\right\}, \\
& I(u ; \eta)=\iint(-\eta L[u])+\int \eta\left\{\frac{\partial u}{\partial n}+\sigma u\right\}
\end{aligned}
$$

folgen daraus die Gleichungen (1) und (1a) bzw. (1b). Wie in [3] arbeiten wir ausschliesslich mit den Gleichungen (6), welche nur die ersten Ableitungen von $u$ und $v$ enthalten.

Das (5) entsprechende finite Problem für eine Bilinearform in $2 n$ Variablen $u_{1}, \ldots, u_{n}, v_{1}, \ldots, v_{n}$ ist elementar lösbar (vergl. [3]). Wir führen den transzendenten Fall auf diesen finiten zurück, indem wir uns zweier geeigneter vollständiger Funktionensysteme $\varphi^{k}$ und $\psi^{i}$ bedienen und z. B. mit dem Ansatz

$$
u=\sum_{k=1}^{n} u_{k} \varphi^{k}, \quad v=\sum_{i=1}^{n} v_{i} \psi^{i}
$$

in die Form $I(u ; v)$ hineingehen, wodurch die finite Form

$$
I(u ; v)=I\left(\sum_{k=1}^{n} u_{k} \phi^{k} ; \sum_{i=1}^{n} v_{i} \psi^{i}\right)=\sum_{k=1}^{n} \sum_{i=1}^{n} I_{i k} v_{i} u_{k}, \quad I_{i k}=I\left(\phi^{k} ; \psi^{i}\right)
$$

entsteht. Die $\varphi^{k}$ und $\psi^{i}$ sind die Eigenfunktionen eines gewöhnlichen "Courantschen Variationsproblems», das zu der quadratischen Integralform $E[u, u]$ gehört, wo $E[u, v]$ der symmetrische Teil von $I(u ; v)$ ist:

$$
E[u, v]=\frac{1}{2}\{I(u ; v)+I(v ; u)\}
$$

Im Gegensatz zu [3] müssen wir hier zwei solche Variationsprobleme lösen, nämlich für jede Randbedingung eines: die $\varphi^{k}$ werden der Bedingung (1a), die $\psi^{i}$ der Bedingung (lb) genügen. Dabei zeigt sich aber, dass der so 
naheliegende Ansatz (7), bei welchem $u$ und $v$ im vornhinein die geforderten Randbedingungen erfüllen, in dieser Form nicht durchführbar ist, weil dann die Abschätzung gewisser Integrale Schwierigkeiten macht. Wir verzichten daher auf eine Randbedingung, und mach€n demgemäss an Stelle von (7) entweder den $" \varphi-\varphi$-Ansatz»

$$
u=\sum_{k=1}^{n} u_{k} \varphi^{k}, \quad v=\sum_{i=1}^{n} v_{i} \varphi^{i}
$$

mit den Variablen $u_{k}, v_{i}$ oder dual dazu den $» \psi$ - $\psi$-Ansatz»

$$
u=\sum_{k=1}^{n} x_{k} \psi^{k}, \quad v=\sum_{i=1}^{n} y_{i} \psi^{i}
$$

mit den Variablen $x_{k}, y_{i}$. Wie man daraus dann neue Paare $(u, v)$ von Funktionen gewinnen kann, bei denen sowohl $u$ als auch $v$ der richtigen Randbedingung genügt, wird sich im Laufe der Arbeit ganz von selbst ergeben. Bei der weiteren Durchführung halten wir uns zunächst an den $\varphi$ - $\varphi$-Ansatz (8), der uns fast Alles, was wir brauchen, liefern wird; jedoch müssen wir an einer späteren Stelle auch noch den $\psi$ - $\psi$-Ansatz berücksichtigen.

\section{Formale Beziehungen und Ungleichungen}

4. Die integrale Bilinearform $I(u ; v)$ baut sich aus einem symmetrischen Anteil $E[u, v]=E[v, u]$ und einem schiefsymmetrischen Anteil $j(u ; v)=$ $-j(v ; u)$ auf. Mit der Abkürzung

$$
c^{*}=c-\frac{1}{2}\left(a_{x}+b_{y}\right)
$$

lauten diese Anteile

$$
\begin{aligned}
& E[u, v]=\iint\left(u_{x} v_{x}+u_{y} v_{y}-c^{*} u v\right)+\frac{1}{2} \int(\sigma+\tau) u v, \\
& j(u ; v)=-\frac{1}{2} \iint\left\{a\left(v u_{x}-u v_{x}\right)+b\left(v u_{y}-u v_{y}\right)\right\},
\end{aligned}
$$

und es ist

$$
I(u ; v)=E[u, v]+j(u ; v) .
$$

In $j(u ; v)$ können wir durch partielle Integration erreichen, dass entweder die Ableitungen von $v$ oder die von $u$ nicht auftreten: im ersten Fall erscheinen die Ableitungen auf $u$ "verlagert», im zweiten Fall auf $v$. 
Wir bezeichnen diese Formen mit $j_{1}(u ; v)$ bzw. $j_{2}(u ; v)$ und ihre flächenhafte Beiträge durch $j_{1}^{*}(u ; v)$ bzw. $j_{2}^{*}(u ; v)$. Es ergibt sich

$$
\begin{aligned}
& j_{1}^{*}(u ; v)=-\iint\left\{a v u_{x}+b v u_{y}+\frac{1}{2}\left(a_{x}+b_{y}\right) v u\right\}, \\
& j_{2}^{*}(u ; v)=\iint\left\{a u v_{x}+b u v_{y}+\frac{1}{2}\left(a_{x}+b_{y}\right) u v\right\}, \\
& j_{1}(u ; v)=j_{1}^{*}(u ; v)+\frac{1}{2} \int(\sigma-\tau) v u, \\
& j_{2}(u ; v)=j_{2}^{*}(u ; v)-\frac{1}{2} \int(\sigma-\tau) u v .
\end{aligned}
$$

Führt man in (12) für $j(u ; v)$ entweder $j_{1}(u ; v)$ oder $j_{2}(u ; v)$ ein, so ergibt sich

$$
\begin{aligned}
& I(u ; v)=\left\{E[u, v]+\frac{1}{2} \int(\sigma-\tau) u v\right\}+j_{1}^{*}(u ; v), \\
& I(u ; v)=\left[E[u, v]-\frac{1}{2} \int(\sigma-\tau) u v\right]+j_{2}^{*}(u ; v) .
\end{aligned}
$$

Für die Ausdrücke in der geschweiften bzw. eckigen Klammer benutzen wir im folgenden die Abkürzungen

$$
\begin{aligned}
\{u, v\} & =E[u, v]+\frac{1}{2} \int(\sigma-\tau) u v= \\
& =\iint\left(u_{x} v_{x}+u_{y} v_{y}-c^{*} u v\right)+\int \sigma v u, \\
{[u, v] } & =E[u, v]-\frac{1}{2} \int(\sigma-\tau) u v= \\
& =\iint\left(u_{x} v_{x}+u_{y} v_{y}-c^{*} u v\right)+\int \tau u v .
\end{aligned}
$$

Es gilt dann

$$
\begin{aligned}
& I(u ; v)=\{u, v\}+j_{1}^{*}(u ; v), \\
& I(u ; v)=[u, v]+j_{2}^{*}(u ; v) .
\end{aligned}
$$

5. Es sei $C>0$ eine Konstante derart, dass im abgeschlossenen Gebiet bzw. auf dem Rand 


$$
\left.\begin{array}{l}
|a|,|b|,|c|,\left|c^{*}\right|, \frac{1}{2}\left|a_{x}+b_{y}\right| \\
\left|a_{x}+b_{y}-c\right|,|\sigma-\tau|
\end{array}\right\} \leqq C
$$

gilt. Dann lassen $\operatorname{sich} j_{1}^{*}(u ; v), j_{2}^{*}(u ; v)$ und $j(u ; v)$ wie folgt abschätzen:

$$
\begin{aligned}
& \left|j_{1}^{*}(u ; v)\right| \leqq C[2 \sqrt{D[u] H[v]}+\sqrt{H[u] H[v]}], \\
& \left|j_{2}^{*}(u ; v)\right| \leqq C[2 \sqrt{H[u] D[v]}+\sqrt{H[u] H[v]}], \\
& |j(u ; v)| \leqq C[\sqrt{H[v] D[u]}+\sqrt{H[u] D[v]}] .
\end{aligned}
$$

Zur Abschätzung der Randintegrale dient die wichtige Ungleichung von Courant, gültig für jєde im abgeschlossenen Gebiet stetige Funktion $f \in \Omega$,

$$
\int f^{2} \leqq C_{1} \sqrt{H[f] D[f]}+C_{2} H[f] ;
$$

hier sind $C_{1}>0$ und $C_{2}>0$ Gebietskonstante; sie gilt z.B. immer dann, wenn der Rand stückweise glatt ist und keine Spitzen aufweist (treten Spitzen auf, so gilt sie, wie man durch einfache Gegenbeispiele belegen kann, nicht mehr). Wir beachten, dass die rechte Seite den Faktor $\sqrt{H[f]}$ enthält. In den späteren Anwendungen handelt es sich oft um die Abschätzung eines Randintegrals $\int f g$, welches zunächst nach der Schwarzschen Ungleichung

$$
\left|\int f g\right| \leqq \sqrt{\int f^{2} \int g^{2}}
$$

abgeschätzt wird; anschliessend wird jeder Faktor rechts gemäss (24) abgeschätzt. Da diese Abschätzung im folgenden häufig auftritt, verweisen wir auf sie kurz als »Standardabschätzung».

\section{Energieintegrale}

6. Wir bezeichnen jeden quadratischen Integralausdruck mit dem » ührungsintegral»

$$
D[f]=\iint\left\{\left(f_{x}\right)^{2}+\left(f_{y}\right)^{2}\right\}
$$

als »Energieintegral», also z. B. die Ausdrücke $E[f, f],\{f, f\},[f, f]$, sowie die entsprechenden Ausdrücke, wenn man den Randbeitrag unterdrückt. Die Courantsche Ungleichung zeigt, dass in einem Energieintegral tatsächlich der durch $D[f]$ gegebene Beitrag »führt». Für später notieren wir die folgenden Eigenschaften: 
1) Für die Elemente $f \in E$, wo $E$ den durch (4) definierten Raum bedeutet, ist ein Energieintegral gleichgradig beschränkt, d.h. es existiert z. B. im Falle von $E[f, f]$ eine für diesen Ausdruck absolute Konstante $C^{*}>0$, so dass

$$
|E[f, f]| \leqq C^{*}
$$

ist. Für den zugehörigen flächenhaften Ausdruck $E^{*}[f, f]$ folgt eine solche Schranke sofort aus der Voraussetzung, dass $H[f] \leqq \Gamma_{1}, D[f] \leqq \Gamma_{2}$ ist, nach dem Muster der Abschätzungen für $j_{1}^{*}(u ; v)$ und $j_{2}^{*}(u ; v)$; für den Randbeitrag ergibt sie sich aus der Courantschen Ungleichung.

2) Mit $H$ bezeichnen wir den Funktionenraum $(f \in \Omega)$

$$
H=\{f \mid H[f] \leqq \Gamma, D[f]<\infty\},
$$

wo $\Gamma>0$ eine absolute Konstante ist. Es ist dann jedes Energieintegral für ein $f \in H$ nach unten beschränkt, was zum Beispiel für $I(f ; f)$ bedeutet: es existiert eine für dieses Integral absolute Konstante $K>0$ derart, dass

$$
I(f ; f) \geqq-2 K
$$

für alle $f \in H$ ist. Der Beweis ist in der folgenden Tatsache begründet: wird ein Energieintegral, für welches $H[f] \leqq \Gamma$ ist, absolut beliebig gross, so entscheidet darüber allein das Führungsglied, was bedeutet, dass das Integral nur nach der positiven Seite beliebig stark anwachsen kann. Der strenge Beweis für die Existenz einer Konstanten $K>0$, für welche (26) gilt, folgt am raschesten indirekt: im Gegenfall existiert eine Folge $f^{n}$ mit $I\left(f^{n} ; f^{n}\right) \rightarrow-\infty$; wegen $H\left[f^{n}\right] \leqq \Gamma$ ist dann notwendig $D\left[f^{n}\right]$ nicht nach oben beschränkt, also für eine Teilfolge $D\left[f^{n}\right] \rightarrow+\infty$; daraus folgt aber $I\left(f^{n} ; f^{n}\right) \rightarrow+\infty$ im Widerspruch zur Annahme, dass $I\left(f^{n} ; f^{n}\right)$ $\rightarrow-\infty$. Dabei wurden implizit wieder die obigen Abschätzungen und die Courantsche Ungleichheit benutzt.

3) Für den Funktionenraum $H$ in (25) gelten Abschätzungen folgender Art für jedes Energieintegral, wobei wir als Beispiel wieder $I(f ; f)$ wählen:

$$
\left\{\begin{aligned}
D[f] & \leqq k^{*}|I(f ; f)|, \\
|I(f ; f)| & \leqq K^{*} D[f] ;
\end{aligned}\right.
$$

hier sind $k^{*}>0, K^{*}>0$ zwei für das betreffende Energieintegral absolute Konstante. Durchläuft $f$ eine Folge $f^{n}$ mit $D\left[f^{n}\right] \rightarrow \infty$, so gilt sogar

$$
D\left[f^{n}\right] \cong I\left(f^{n} ; f^{n}\right) \quad(\text { für } n \rightarrow \infty) .
$$

Um nicht immer die dabei auftretenden Konstanten mitführen zu müssen, schreiben wir später im Falle einer Folge $f^{n}$ anstelle von $H\left[f^{n}\right] \leqq \Gamma$ kurz $H\left[f^{n}\right]=O(1)$ und für (27a) entsprechend 


$$
\begin{aligned}
D\left[f^{n}\right] & =O\left(\left|I\left(f^{n} ; f^{n}\right)\right|\right), \\
I\left(f^{n} ; f^{n}\right) & =O\left(D\left[f^{n}\right]\right) .
\end{aligned}
$$

4) Beschränken wir uns auf Folgen $f^{n}$, so lautet in neuer Schreibung das Ergebnis unter 1): aus $H\left[f^{n}\right]=O(1)$ und $D\left[f^{n}\right]=O(1)$ folgt für jedes Energieintegral z. B. $\{f, f\}$, dass auch $\left\{f^{n}, f^{n}\right\}=O(1)$ ist. Nach 3) gilt davon die folgende Umkehrung: aus $H\left[f^{n}\right]=O(1)$ und $\left\{f^{n}, f^{n}\right\}=O(1)$ folgt, dass auch $D\left[f^{n}\right]=O(1)$ ist. Wir deuten den dadurch gegebenen Sachverhalt unmissverständlich so an:

$$
\left.\begin{array}{l}
H\left[f^{n}\right]=O(1) \\
D\left[f^{n}\right]=O(1)
\end{array}\right\} \leftrightarrow\left\{\begin{array}{r}
H\left[f^{n}\right]=O(1) \\
\left\{f^{n}, f^{n}\right\}=O(1)
\end{array} .\right.
$$

5) In der vorigen Schreibung gilt ganz entsprechend die Doppelaussage:

$$
\left.\begin{array}{l}
H\left[f^{n}\right]=o(1) \\
D\left[f^{n}\right]=o(1)
\end{array}\right\} \rightleftarrows\left\{\begin{array}{r}
H\left[f^{n}\right]=o(1) \\
\left\{f^{n}, f^{n}\right\}=o(1)
\end{array},\right.
$$

und analog für jedes andere Energieintegral anstelle von $\{f, f\}$. Der Beweis in der Richtung von links nach rechts ergibt sich sofort. In der umgekehrten Richtung erfolgt der Beweis so: nach 4) ist $D\left[f^{n}\right]=O(1)$, also wegen $H\left[f^{n}\right]=o(1)$ nach der Courantschen Ungleichung $\int \sigma\left(f^{n}\right)^{2}=$ $o(1)$; in

$$
\left\{f^{n}, f^{n}\right\}=\iint\left\{\left(f_{x}^{n}\right)^{2}+\left(f_{y}^{n}\right)^{2}\right\}-\iint c^{*}\left(f^{n}\right)^{2}+\int \sigma\left(f^{n}\right)^{2}
$$

sind die linke Seite und die zwei letzten Integrale auf der rechten Seite ein $o(1)$ und folglich auch $D\left[f^{n}\right]=o(1)$. Wir erinnern daran, dass in der Schreibung von $\S 2$ die betreffende Folge $f^{n}$ äquivalent 0 i. Z. $f^{n} \sim 0$ ist.

Wir vereinbaren noch folgende Sprechweise: gilt für alle Funktionen $f$ aus dem Raum $(f \in \Omega)$

$$
H=\{f \mid H[f] \leqq \Gamma, D[f]<\infty\}
$$

und ein Energieintegral, z. B. $I(f ; f)$, eine Abschätzung

$$
|I(f ; f)| \leqq C^{*}
$$

( $C^{*}>0$ eine nur von dem Raum $H$, jedoch nicht von dem einzelnen Element $f$ abhängige absolute Konstante), so sagen wir: die betreffenden Funktionen sind »energiebeschränkt». 4) zeigt, dass dann die Funktionen $f$ einem Raum $E$, wie er in $\S 2$ (4) definiert ist, angehören, und 1) zeigt, dass umgekehrt alle $f$ aus einem Raum $E$ auch energiebeschränkt sind. 


\section{Die beiden zugeordneten Courantschen Variationsprobleme}

7. $\mathrm{Zu}$ der integralen Bilinearform $I(u ; v)$ gehört die symmetrische Form $E[u, v]$ und zu dieser die quadratische Integralform $E[u, u]$. Auf diese Integralform lässt sich dann nach dem Vorbild von Courant eine übliche Variationsaufgabe gründen, in welcher sukzessive in wachsender Reihenfolge Minima auftreten. Im Gegensatz zu unserer früheren Arbeit [3] müssen wir aber jetzt entsprechend den zwei Randbedingungen (la) und $(1 \mathrm{~b})$ diese Variationsaufgabe zweimal ansetzen: einmal für die erste Randbedingung, das andere Mal für die zweite Randbedingung. Wir erreichen dies, indem wir jeweils noch ein passendes Randintegral hinzunehmen, wodurch das betr. Variationsproblem ein freies wird. Es sind dies die in $\S 4$ durch (17) bzw. (18) gegebenen Ausdrücke, spezialisiert für $v=u$ bzw. $u=v$ :

$$
\begin{aligned}
& \{u, u\}=E[u, u]+\frac{1}{2} \int(\sigma-\tau) u^{2} \\
& {[v, v]=E[v, v]-\frac{1}{2} \int(\sigma-\tau) v^{2} .}
\end{aligned}
$$

Wir gelangen so zu zwei zugeordneten Courantschen Variationsproblemen I und II, die wir sogleich genau formulieren. Wir dürfen und wollen diese Probleme, welche naturgemäss viel einfacher sind als das ursprüngliche Problem für die Form $I(u ; v)$, als gelöst ansehen, und werden dann mit ihrer Hilfe das Problem für $I(u ; v)$ lösen. Das bedingt, dass wir für die Koeffizientenfunktionen $a, b, c(a=a(x, y), \ldots)$ die Voraussetzungen als erfüllt annehmen müssen, die in Courant-Hilbert [2], Seite 476, stehen. Für das folgende vergleiche man auch Courant-Hilbert [1], Seite $345 \mathrm{ff}$.

8. Problem I. Es sei $\varphi \in \Omega$ und die quadratische Integralform vorgelegt:

$$
\begin{aligned}
\{\varphi, \varphi\} & =E[\varphi, \varphi]+\frac{1}{2} \int(\sigma-\tau) \varphi^{2} \\
& =\iint\left\{\left(\varphi_{x}\right)^{2}+\left(\varphi_{y}\right)^{2}-c^{*} \varphi^{2}\right\}+\int \sigma \varphi^{2} .
\end{aligned}
$$

Aufgabe $I_{1}$ : Unter allen Funktionen $\varphi \in \Omega$, welche der quadratischen Nebenbedingung

$$
H[\varphi, \varphi]=\|\varphi\|^{2}=1
$$


genügen, ist eine solche gesucht, für welche der Ausdruck $\{\varphi, \varphi\}$ ein Minimum wird. Aus $\S 6$ Absatz 2) und speziell aus der Beziehung (26) geht hervor, dass diese Aufgabe sinnvoll ist, und dass jedenfalls eine untere Grenze für den Ausdruck $\{\varphi, \varphi\}$ existiert. Und da wir, wie oben gesagt, diese und ebenso die folgenden Aufgaben als lösbar und gelöst ansehen dürfen, so wird die untere Grenze $x_{1}$ von einer Funktion $\varphi^{1} \in \Omega$ sogar angenommen. Für die minimierende Funktion $\varphi^{1}$ ergibt sich dann sofort die folgende noch nicht ausintegrierte Variationsgleichung

$$
\left\{\varphi^{1}, \zeta\right\}-\varkappa_{1} H\left[\varphi^{1}, \zeta\right]=0,
$$

gültig für eine beliebige Funktion $\zeta \in \Omega$. Speziell folgt aus (28) für $\zeta=q^{1}$ wegen $\left\|\varphi^{1}\right\|^{2}=1$, dass $\left\{\varphi^{1}, \varphi^{1}\right\}=\varkappa_{1}$ ist. Nehmen wir darüber hinaus an, dass $\varphi^{1}$ im Gebiet zweimal stetig differenzierbar ist, und dass auf dem Rand der Ausdruck $\partial \varphi^{1} / \partial n+\sigma \varphi^{1}$ existiert, so folgt aus (28), wenn man die Ableitungen von $\zeta$ vertreibt, für $\varphi^{1}$ die Differentialgleichung nebst Randbedingung

$$
\Delta \varphi^{1}+c^{*} \varphi^{1}+\varkappa_{1} \varphi^{1}=0, \frac{\partial \varphi^{1}}{\partial n}+\sigma \varphi^{1}=0,
$$

das Erfülltsein der Randbedingung im Sinne von Courant zu verstehen. Indessen brauchen wir im folgenden dieses Resultat nicht, sondern nur die nichtausintegrierte Variationsgleichung (28) unter der Annahme, dass $\varphi^{1}$ existiert und $\varphi^{1} \in \Omega$ ist. Wir drücken (28) kurz dahin aus, dass wir sagen: $\varphi^{1}$ genügt der betreffenden Variationsgleichung und im Hinblick auf (29) der vorgeschriebenen Randbedingung (1a). Nachdem so $x_{1}$ und $\varphi^{1}$ bekannt sind, kommen wir zur

Aufgabe $I_{2}$ : Unter allen Funktionen $\varphi \in \Omega$, welche der quadratischen Nebenbedingung

$$
H[\varphi]=\| \psi^{\prime 2}=1
$$

und der linearen Nebenbedingung

$$
H\left[\varphi^{1}, \varphi\right]=0
$$

genügen, ist eine solche gesucht, für welche der Ausdruck $\{\varphi, \varphi\}$ ein Minimum wird. Wie bei der Aufgabe $I_{1}$ existiert hier ein IIinimum $\varkappa_{2}$ und eine zugehörige minimierende Funktion $\varphi^{2}$ wobei wegen (30) notwendig $x_{1} \leqq x_{2}$ ist. Und für $\varphi^{2}$ gilt die zu (28) analoge Variationsgleichung

$$
\left\{\varphi^{2}, \zeta\right\}-\varkappa_{2} H\left[\varphi^{2}, \zeta\right]=0,
$$

$\zeta$ beliebig aus $\Omega$. Für $\zeta=\varphi^{2}$ folgt speziell $\left\{\varphi^{2}, \varphi^{2}\right\}=\varkappa_{2}$. Ebenso gilt die zu (29) analoge Differentialgleichung nebst Randbedingung, Alles zu verstehen in dem oben präzisierten Sinne. Kurz gesagt: $\varphi^{2}$ genügt der 
Variationsgleichung (31) und der Randbedingung (1a). Wegen der linearen Nebenbedingung gilt

$$
H\left[\varphi^{1}, \varphi^{2}\right]=0,
$$

i. W.: $\varphi^{2}$ ist "orthogonal» zu $\varphi^{1}$. Ferner folgt aber aus (31) für $\zeta=\varphi^{2}$ wegen $H\left[\varphi^{1}, \varphi^{2}\right]=0$, dass auch $\left\{\varphi^{1}, \varphi^{2}\right\}=0$ ist.

Es ist klar, wie das Verfahren weitergeht: beim nächsten Schritt unterwirft man $\varphi$ ausser der Bedingung $H[\varphi]=\|\varphi\|^{2}=1$ noch den zwei linearen Nebenbedingungen $H\left[\varphi^{1}, \varphi\right]=0$ und $H\left[\varphi^{2}, \varphi\right]=0$ und erhält so ein neues Minimum $\varkappa_{3}$ und eine neue Funktion $\varphi^{3}$, und so fort. Das Verfahren bricht nicht ab und liefert eine nichtfallende Folge von Eigenwerten

$$
x_{1} \leqq x_{2} \leqq x_{3} \leqq \cdots
$$

mit zugehörigen Eigenfunktionen

$$
\varphi^{1}, \varphi^{2}, \varphi^{3}, \cdots .
$$

Dabei gelten die Variationsgleichungen für $i=1,2, \ldots$

$$
\left\{\varphi^{i}, \zeta\right\}-\varkappa_{i} H\left[\varphi^{i}, \zeta\right]=0
$$

für jedes $\zeta \in \Omega$, sowie die Aussagen:

$$
\begin{gathered}
H\left[\varphi^{i}, \varphi^{k}\right]=\left\{\begin{array}{lll}
1 & \text { für } i=k \\
0 & \text { für } i \neq k
\end{array},\right. \\
\left\{\varphi^{i}, \varphi^{k}\right\}=\left\{\begin{array}{lll}
\varkappa_{i} & \text { für } i=k \\
0 & \text { für } i \neq k
\end{array} ;\right.
\end{gathered}
$$

schliesslich genügt $\varphi^{i}$ der Randbedingung (1a) im Courantschen Sinne

$$
\frac{\partial \varphi^{i}}{\partial n}+\sigma \varphi^{i}=0 \text {. }
$$

Weiter gilt, was wir ohne Beweis übernehmen: die Eigenwerte $\varkappa_{p}$ wachsen unbegrenzt an, genauer, es gilt für sie die Lorentz-Sommerfeldsche Vermutung

$$
\varkappa_{p} \cong \frac{4 \pi p}{F}
$$

für $p \rightarrow \infty$.

9. Problem II. Nach dem Vorbild des vorigen Paragraphen können wir uns hier kürzer fassen. Es sei $\psi \in \Omega$ und die quadratische Integralform vorgelegt: 


$$
\begin{aligned}
{[\psi, \psi] } & =E[\psi, \psi]-\frac{1}{2} \int(\sigma-\tau) \psi^{2} \\
& =\iint\left\{\left(\psi_{x}\right)^{2}+\left(\psi_{y}\right)^{2}-c^{*} \psi^{2}\right\}+\int \tau \psi^{2} .
\end{aligned}
$$

Aufgabe $I I_{1}$ : Unter allen Funktionen $\psi \in \Omega$, welche der Normierungsbedingung

$$
H[\psi]=\|\psi\|^{2}=1
$$

genügen, ist eine solche gesucht, für welche der Ausdruck $[\psi, \psi]$ ein Minimum wird. Wie bei der Aufgabe $I_{1}$ existiert ein Minimum $\mu_{1}$ und eine zugehörige minimierende Funktion $\psi^{\mathbf{1}}$. Die Funktion $\psi^{\mathbf{1}}$ genügt der Variationsgleichung

$$
\left[\psi^{1}, \eta\right]-\mu_{1} H\left[\psi^{1}, \eta\right]=0
$$

für jedes $\eta \in \Omega . \quad \eta=\psi^{1}$ liefert $\left[\psi^{1}, \psi^{1}\right]=\mu_{1}$. Ferner genügt unter entsprechenden Differenzierbarkeitsannahmen $\psi^{1}$ der Differentialgleichung nebst Randbedingung

$$
\Delta \psi^{1}+c^{*} \psi^{1}+\mu_{1} \psi^{1}=0, \quad \frac{\partial \psi^{1}}{\partial n}+\tau \psi^{1}=0 .
$$

Kurz: $\psi^{1}$ genügt der zugehörigen Variationsgleichung (38) und der zweiten Randbedingung (1b), zu verstehen im Courantschen Sinne.

Aufgabe $I I_{2}$ : Hier wird die analoge Aufgabe wie bei $I I_{1}$ gestellt mit dem Zusatz: $\psi$ hat ausser der Normierungsbedingung $\|\psi\|^{2}=1$ noch der linearen Nebenbedingung $H\left[\psi^{1}, \psi\right]=0$ zu genügen. Das liefert den nächsten Eigenwert $\mu_{2}$, wo $\mu_{1} \leqq \mu_{2}$ ist, und die nächste Eigenfunktion $\psi^{2}$, wobei $\left[\psi^{2}, \psi^{2}\right]=\mu_{2}$ ist; ferner gilt $H\left[\psi^{1}, \psi^{2}\right]=0$ und $\left[\psi^{1}, \psi^{2}\right]=0$.

Und wie beim Problem I ergibt sich eine nichtfallende Folge von Eigenwerten

$$
\mu_{1} \leqq \mu_{2} \leqq \mu_{3} \leqq \cdots
$$

nebst zugehörigen Eigenfunktionen

$$
\psi^{1}, \psi^{2}, \psi^{3}, \cdots
$$

Dabei gelten die Variationsgleichungen für $i=1,2, \cdots$

$$
\left[\psi^{i}, \eta\right]-\mu_{i} H\left[\psi^{i}, \eta\right]=0
$$

für jedes $\eta \in \Omega$, sowie die Aussagen:

$$
\begin{gathered}
H\left[\psi^{i}, \psi^{k}\right]=\left\{\begin{array}{lll}
1 & \text { für } & i=k \\
0 & \text { für } & i \neq k
\end{array}\right. \\
{\left[\psi^{i}, \psi^{k}\right]=\left\{\begin{array}{lll}
\mu_{i} & \text { für } & i=k \\
0 & \text { für } & i \neq k
\end{array}\right.}
\end{gathered}
$$


schliesshıch genügt $\psi^{i}$ der Randbedingung (lb) im Courantschen Sinne

$$
\frac{\partial \psi^{i}}{\partial n}+\tau \psi^{i}=0 \text {. }
$$

Weiter gilt auch hier wie beim Problem I für die Eigenwerte $\mu_{p}$ dasselbe asymptotische Gesetz (Lorentz-Sommerfeldsche Vermutung) wie für die $\varkappa_{p}$

$$
\mu_{p} \cong \frac{4 \pi p}{F}
$$

für $p \rightarrow \infty$.

Dass in beiden Fällen für die Eigenwerte dieselbe asymptotische Formel gilt, bringt lediglich die bekannte Tatsache zum Ausdruck, dass Randbedingungen keinen Einfluss auf das asymptotische Verhalten der zugehörigen Eigenwerte ausüben.

10. Die beiden Tatsachen

1) die Probleme I und II sind freie Probleme (ohne Randbedingungen)

2) die Eigenwerte wachsen über alle Grenzen $\left(\kappa_{n} \rightarrow \infty\right.$ und $\mu_{n} \rightarrow \infty$ für $n \rightarrow \infty$ ) ziehen bekanntlich nach sich, dass z. B. beim Problem I (analog ist es beim Problem II) Folgendes gilt:

Für eine Funktion $f \in \Omega$ mit den $»$ Fourier-Koeffizienten»

$$
a_{i}=H\left[f, \varphi^{i}\right]
$$

und der zugehörigen Fourier-Reihe (das Zeichen $\sim$ bedeutet in diesem Zusammenhang eine Zuordnung)

$$
f \sim \sum_{i=1}^{\infty} a_{i} \varphi^{i}
$$

gelten, wenn

$$
f_{n}=f-\sum_{i=1}^{\infty} a_{i} \psi^{i}
$$

gesetzt wird, die sogenannten Vollständigkeitsrelationen

$$
H\left[f_{n}\right] \rightarrow 0, \quad\left\{f_{n}, f_{n}\right\} \rightarrow 0
$$

für $n \rightarrow \infty$. Gleichbedeutend damit sind die Aussagen

$$
\begin{gathered}
H[f]=\sum_{i=1}^{\infty} a_{i}^{2}, \\
\{f, f\}=\sum_{i=1}^{\infty} x_{i} a_{i}^{2} .
\end{gathered}
$$


Von diesen gilt die erste Gleichung für jedes stückweise stetige $f$ mit $H[f]<\infty$.

Eine Folgerung aus der Relation (41b) wird später benutzt werden: denken wir uns die Relation (41b) für alle $f$ aus dem Funktionenraum $E$ in (4) gebildet, so ergibt sich aus dem in $\S 6$ unter 1) über Energieintegrale Gesagten, dass eine Abschätzung

$$
\sum_{v=m+1}^{\infty} a_{v}^{2} \leqq M \varkappa_{m+1}^{-1}
$$

"gleichgradig" (d.h. die Konstante $M>0$ ist von dem einzelnen $f \in E$ unabhängig) gilt.

\section{Ansatz zur Lösung der Aufgabe}

11. Wir gehen mit dem $» \varphi-\varphi$-Ansatz»

$$
u=\sum_{k=1}^{n} u_{k} \phi^{k}, \quad v=\sum_{i=1}^{n} v_{i} \varphi^{i},
$$

wo die Funktionen $\varphi^{i}$ dem Variationsproblem I entspringen, in die Form $I(u ; v)$ hinein und erhalten die finite Bilinearform in den $2 n$ Variablen $u_{1}, \ldots, u_{n}$ und $v_{1}, \ldots, v_{n}$ :

$$
I(u ; v)=\sum_{k=1}^{n} \sum_{i=1}^{n} I_{i k} v_{i} u_{k}
$$

mit $I_{i k}=I\left(\phi^{k} ; \varphi^{i}\right)$. Wir fassen diese Form als quadratische Form ihrer Variablen in einem $2 n$-dimensionalen Raum $R^{2 n}$ auf: dann gehört zu ihr ein Spektrum von Eigenwerten mit zugehörigen Eigenvektoren, deren Verhalten für $n \rightarrow \infty$ wir studieren. Dabei normieren wir die Eigenvektoren in Übereinstimmung mit der Normierung $\|u\|^{2}+\|v\|^{2}=2$ in (5) gemäss

$$
\sum_{k=1}^{n} u_{k}^{2}+\sum_{i=1}^{n} v_{i}^{2}=2 .
$$

Wir vereinbaren die folgende Schreibung, wobei wir die Abhängigkeit von $n$ mit ausdrücken. Das Spektrum der Eigenwerte sei nach wachsender Grösse durch die Zahlen

$$
-\lambda_{n}^{n} \leqq-\lambda_{n}^{n-1} \leqq \cdots \leqq-\lambda_{1}^{n} \leqq \lambda_{1}^{n} \leqq \lambda_{2}^{n} \leqq \cdots \leqq \lambda_{n}^{n}
$$

gegeben; es ist symmetrisch zum Ursprung, da mit einem $\lambda$ stets auch $-\lambda$ Eigenwert ist, und liegt vollständig vor, wenn $\operatorname{man} \lambda_{1}^{n}$ bis $\lambda_{n}^{n}$ kennt, die alle $\geqq 0$ sind. Zu dem Eigenwert $\lambda_{p}^{n}, \lambda_{p}^{n} \geqq 0$, gehört ein Eigenvektor, den wir so schreiben 


$$
U_{p}^{n}=\sum_{k=1}^{n} U_{p k}^{n} \varphi^{k}, \quad V_{p}^{n}=\sum_{i=1}^{n} V_{p i}^{n} \varphi^{i}
$$

und den wir zu dem Paar

$$
\left(U_{p}^{n}, V_{p}^{n}\right)
$$

zusammenfassen, wobei also diese Klammer den $2 n$-dimensionalen Vektor mit den Koordinaten

$$
U_{p 1}^{n}, \ldots, U_{p n}^{n}, V_{p 1}^{n}, \ldots, V_{p n}^{n}
$$

»darstellt». Dabei sprechen wir das Funktionenpaar je nach Lage als »Eigenfunktion» oder als "Eigenvektor» an; ein Missverständnis ist aus dem jeweiligen Zusammenhang ausgeschlossen. Es gehört dann $\mathrm{zu}-\lambda_{p}^{n}$ der Eigenvektor

$$
\left(U_{p}^{n},-V_{p}^{n}\right)
$$

ebenfalls mit dem Normquadrat 2. Mit dieser Normierung bilden die $2 n$ Eigenvektoren ein orthonormiertes und vollständiges Vektorsystem des Raumes $R^{2 n}$. Ferner gilt wegen der Orthogonalität der beiden Vektoren (45) und (46), dass

$$
\sum_{k=1}^{n}\left(U_{p k}^{n}\right)^{2}=\sum_{i=1}^{n}\left(V_{p i}^{n}\right)^{2}=1
$$

ist, und weiter nach [3], dass die $n$-dimensionalen Vektoren $U_{p}^{n}$ bzw. $V_{p}^{n}$ bereits je für sich ein vollständiges und zwar jetzt mit der Norm 1 normiertes Vektorsystem im $n$-dimensionalen Raum der $\varphi^{1}, \ldots, \varphi^{n}$ bilden. Da das System $\varphi^{i}$ orthonormiert ist, so ist auch z. B.

$$
\left\|U_{p}^{n}\right\|^{2}=H\left[U_{p}^{n}\right]=\sum_{k=1}^{n}\left(U_{p k}^{n}\right)^{2} .
$$

Ist $\left(\zeta^{n}, \eta^{n}\right)$ ein beliebiger Vektor

$$
\zeta^{n}=\sum_{k=1}^{n} \zeta_{k} \varphi^{k}, \quad \eta^{n}=\sum_{i=1}^{n} \eta_{i} \varphi^{i}
$$

so gelten für einen Eigenvektor die strengen "Variationsgleichungen»:

$$
\begin{aligned}
& I\left(U_{p}^{n} ; \eta^{n}\right)-\lambda_{p}^{n} H\left[V_{p}^{n}, \eta^{n}\right]=0, \\
& I\left(\zeta^{n} ; V_{p}^{n}\right)-\lambda_{p}^{n} H\left[\zeta^{n}, U_{p}^{n}\right]=0 ;
\end{aligned}
$$

aus ihnen folgt ( $\eta^{n}$ durch $V_{q}^{n}$ ersetzt)

$$
I\left(U_{p}^{n} ; V_{q}^{n}\right)=\left\{\begin{array}{lll}
\lambda_{p}^{n} & \text { für } & p=q \\
0 & \text { für } & p \neq q
\end{array}\right.
$$

$(p, q=1, \ldots, n)$. 
In dem Funktionenpaar $\left(U_{p}^{n}, V_{p}^{n}\right)$ ( $p$ fest) haben wir für grosse $n$ approximativ eine "Eigenfunktion» $(u, v)$ für das Variationsproblem (5) für $I(u ; v)$ zu sehen.

Wie schon früher betont, benötigen wir aber nicht die Existenz von Eigenfunktionen $(u, v)$; es genügt vielmehr, dass wir $(u, v)$ durch eine passende Folge von Näherungsfunktionen »ersetzen», welche wir nach dem Vorbild der Schreibung in (45) auch durch

$$
\left(U^{n}, V^{n}\right)
$$

bezeichnen.

\section{Eigenfolgen}

12. Nach dem in $\S 11$ Gesagten ergibt sich die Definition der Eigenfolge für das Variationsproblem (5) für $I(u ; v)$ ganz von selbst. Dabei ist es hier sachgemäss, Alles in der Sprache von Funktionspaaren wie $(U, V)$ oder $(\zeta, \eta)$ auszudrücken, die wir kurz als eine Funktion ansprechen; analog ist $\left(U^{n}, V^{n}\right)$ kurz eine Folge von Funktionen. Naturgemäss werden wir an eine Folge, z. B. $\left(f^{n}, g^{n}\right)$, die Forderung stellen, dass sie zu einem Raum $E$ gehört, wie er früher in (4) definiert wurde, d.h. dass $f^{n} \in \Omega, g^{n} \in \Omega$ und dass $H\left[f^{n}\right]=O(1), H\left[g^{n}\right]=O(1)$ sowie $D\left[f^{n}\right]=O(1)$ und $D\left[g^{n}\right]=$ $O(1)$ ist, von uns kurz ausgedrückt durch

$$
\left(f^{n}, g^{n}\right) \in E \text {. }
$$

Definition: $\lambda \geqq 0$ heisst Eigenwert und $\left(U^{n}, V^{n}\right)$ eine zugehörige Eigenfolge des Variationsproblems

$$
I(u ; v)=\text { stationär }
$$

für alle Funktionen $(u, v) \in \Omega$ mit der Normierung $\|u\|^{2}+\|v\|^{2}=2$, wenn folgende Bedingungen erfüllt sind:

1) Es gilt

$$
\left(U^{n}, V^{n}\right) \in E .
$$

2) Für eine beliebige Folge $\left(\zeta^{n}, \eta^{n}\right)$ mit

$$
\left(\zeta^{n}, \eta^{n}\right) \in E
$$

gelten im Sinne eines $o(1)$ die "Variationsgleichungen»

$$
\begin{aligned}
& I\left(U^{n} ; \eta^{n}\right)-\lambda H\left[V^{n}, \eta^{n}\right]=o(1), \\
& I\left(\zeta^{n} ; V^{n}\right)-\lambda H\left[\zeta^{n}, U^{n}\right]=o(1) .
\end{aligned}
$$

3) Es ist

$$
\left\|U^{n}\right\|^{2}+\left\|V^{n}\right\|^{2}=2+o(1)
$$


Das Weitere sinngemäss wie in [3], Seite 12-15. Wir erinnern kurz an die dort gebrachten Folgerungen und Definitionen.

$1^{\circ}$. Mit der obigen Eigenfolge ist gleichzeitig auch $\left(U^{n},-V^{n}\right)$ Eigenfolge zum negativen Wert $-\lambda$ (in der obigen Definition also $\lambda$ durch $-\lambda$ ersetzt).

$2^{\circ}$. Es ist für $\lambda>0$

$$
\left\|U^{n}\right\|^{2}=1+o(1), \quad\left\|V^{n}\right\|^{2}=1+o(1)
$$

ist aber $\lambda=0$, so können und wollen wir diese Normierung im vornhinein annehmen.

$3^{\circ}$. Der Begriff der »Nullfolge» (statt Nullfunktionenfolge wie in [3]): sie ist in der in $\S 2$ verabredeten Schreibung durch die zwei Forderungen

$$
\left(\Phi^{n}, \Psi^{n}\right) \in E, \quad\left(\Phi^{n}, \Psi^{n}\right) \sim(0,0)
$$

definiert und spielt im Rahmen unserer Betrachtungen die Rolle der Null. Es ist z. B. mit $\left(U^{n}, V^{n}\right)$ stets auch $\left(U^{n}+\Phi^{n}, V^{n}+\Psi^{n}\right)$ Eigenfolge zum gleichen Eigenwert. Gilt in (51) die zweite Beziehung nicht, so schreiben wir $\left(\Phi^{n}, \Psi^{n}\right) \sim(0,0)$; z. B. ist sicher für eine Eigenfolge $\left(U^{n}, V^{n}\right) \sim$ $(0,0)$.

$4^{\circ}$. Die $r+1$ Eigenfolgen $\left(U_{v}^{n}, V_{v}^{n}\right) \quad(v=1,2, \ldots, r+1)$ heissen linear abhängig, wenn Zahlenfolgen $a_{1}^{n}, a_{2}^{n}, \ldots, a_{r+1}^{n}$ mit $\left(a_{1}^{n}\right)^{2}+$ $\cdots+\left(a_{r+1}^{n}\right)^{2}=1+o(1)$ existieren, derart, dass in leichtverständlicher Schreibung

$$
\sum_{\nu=1}^{r+1} a_{\nu}^{n}\left(U_{v}^{n}, V_{\nu}^{n}\right) \sim(0,0)
$$

ist. Im Gegenfall heissen die $r+1$ Eigenfolgen linear unabhängig. Trifft (52) zu, so existiert ein Zeiger $\in\{1,2, \ldots, r+1\}$, als welchen wir $r+1$ wählen dürfen, für welchen eine Teilfolge von $a_{r+1}^{n}$ nach einer Zahl $\neq 0$ konvergiert, und es gilt dann nach geeigneter Aussiebung einer Teilfolge für $n$ mit neuen Zahlenfolgen $b_{1}^{n}, \ldots, b_{r}^{n}$ eine Äquivalenz

$$
\left(U_{r+1}^{n}, V_{r+1}^{n}\right) \sim \sum_{v=1}^{r} b_{\nu}^{n}\left(U_{v}^{n}, V_{v}^{n}\right)
$$

wobei notwendig für die $b_{v}^{n}$ eine Beziehung $0<\beta \leqq\left(b_{1}^{n}\right)^{2}+\cdots+\left(b_{r}^{n}\right)^{2}$ $\leqq B$ mit Konstanten $\beta, B$ gilt, die von $n$ nicht abhängen. Wir sagen für den Sachverhalt (53) kurz: die Eigenfolge $\left(U_{r+1}^{n}, V_{r+1}^{n}\right)$ ist eine lineare Kombination der Eigenfolgen $\left(U_{v}^{n}, V_{v}^{n}\right)$, wo $v$ von 1 bis $r$ läuft, bzw. kürzer $\left(U_{r+1}^{n}, V_{r+1}^{n}\right)$ ist unter den $r$ Eigenfolgen $\left(U_{v}^{n}, V_{v}^{n}\right)$ enthalten.

$5^{\circ}$. Zwei Eigenfolgen $\left(U^{n}, V^{n}\right)$ und $\left(\tilde{U}^{n}, \tilde{V}^{n}\right)$ heissen zueinander orthogonal, wenn 


$$
H\left[U^{n}, \tilde{U}^{n}\right]+H\left[V^{n}, \tilde{V}^{n}\right]=o(1)
$$

ist, und sie heissen einzeln orthogonal zueinander, wenn

$$
H\left[U^{n}, \tilde{U}^{n}\right]=o(1), \quad H\left[V^{n}, \tilde{V}^{n}\right]=o(1)
$$

ist. Z. B. sind zwei Eigenfolgen, die zu verschiedenen Eigenwerten gehören, stets zueinander orthogonal und sogar einzeln zueinander orthogonal.

13. Um auch die triviale Lösung

$$
\left(U^{n}, V^{n}\right) \sim(0,0)
$$

der Variationsgleichungen (50) zu erfassen, geben wir noch die

Definition: $\left(U^{n}, V^{n}\right)$ heisst eine verallgemeinerte Eigenfolge zum Wert $\lambda$, wenn sie die Eigenschaften 1) und 2) einer Eigenfolge hat; die Normierungseigenschaft 3) braucht dagegen nicht erfüllt zu sein.

Unser weiteres Ziel ist es, nachzuweisen, dass für ein festes $p$ und für wachsendes $n$ die in $\S 11$ definierte Folge $\lambda_{p}^{n}$ konvergiert, also $\lim \lambda_{p}^{n}=$ $\lambda_{p} \quad\left(\lambda_{p} \geqq 0\right)$ existiert, dass $\lambda_{p}$ Eigenwert ist, und dass die zu $\lambda_{p}^{n}$ gehörige Folge $\left(U_{p}^{n}, V_{p}^{n}\right)$ eine Eigenfolge zum Wert $\lambda_{p}$ ist.

\section{Erste Abschätzungen für $\lambda_{p}^{n}$ und $\left(U_{p}^{n}, V_{p}^{n}\right)$}

14. Wir knüpfen an $\S 11$ an, und schreiben die dortige finite Bilinearform wie folgt um: es ist nach (19) $I(u ; v)=\{u, v\}+j_{1}^{*}(u ; v)$; trägt man darin die Ausdrücke (43) für $u$ und $v$ ein, und berücksichtigt die Relationen (35) für die $\varphi^{i}$, so bekommt $\{u, v\}$ die $\gg$ Diagonalform»

$$
\sum_{i=1}^{n} \varkappa_{i} u_{i} v_{i}
$$

und wir erhalten

$$
I(u ; v)=\sum_{i=1}^{n} \varkappa_{i} u_{i} v_{i}+j_{1}^{*}\left(\sum_{k=1}^{n} u_{k} \varphi^{k} ; \sum_{i=1}^{n} v_{i} \varphi^{i}\right),
$$

wobei

$$
u=\sum_{k=1}^{n} u_{k} \varphi^{k} \text { und } v=\sum_{i=1}^{n} v_{i} \varphi^{i}
$$

ist. Thr Spektrum ist durch (44) gegeben, wobei nur die positive Hälfte d. h. die Zahlen $0 \leqq \lambda_{1}^{n} \leqq \lambda_{2}^{n} \leqq \cdots \leqq \lambda_{n}^{n}$ interessieren; die zugehörigen »Eigenfunktionen» sind durch die $\left(U_{p}^{n}, V_{p}^{n}\right)$ in (45) gegeben $(p=1, \ldots, n)$. Zur Abschätzung von $\lambda_{p}^{n}$ nach unten benutzen wir die max-min-Eigenschaft, zur Abschätzung nach oben die min-max-Eigenschaft von $\lambda_{p}^{n}$. 
$\left(B_{v}\right)$ bzw. $\left(B_{u}\right)$ bedeutet im folgenden eine lineare homogene Bindung für die "Variablen» $u_{1}, \ldots, u_{n}, v_{1}, \ldots, v_{n}$. Die folgenden Überlegungen verlaufen ganz ähnlich denen in [3], Seite 16-19, so dass wir uns kürzer fassen können.

15. Die max-min-Eigenschaft von $\lambda_{p}^{n}$ besagt: wir bestimmen für $n+p-1$ "Bindungen» $\left(B_{v}\right)$ das Minimum der rechten Seite von (54) bei der Normierung

$$
\|u\|^{2}+\|v\|^{2}=\sum_{k=1}^{n} u_{k}^{2}+\sum_{i=1}^{n} v_{i}^{2}=2 ;
$$

alsdann ist $\lambda_{p}^{n}$ das Maximum dieser Minima für alle möglichen solche Bindungen $\left(B_{v}\right) \quad(v=1,2, \ldots, n+p-1)$. Wir wählen als solche Bindungen

$$
\left(B_{v}\right): \quad u_{1}-v_{1}=0, \ldots, u_{n}-v_{n}=0, u_{1}=0, \ldots, u_{p-1}=0
$$

und erhalten

$$
\lambda_{p}^{n} \geqq \min \left\{\sum_{i=p}^{n} x_{i} u_{i}^{2}+j_{1}^{*}\left(\sum_{k=p}^{n} u_{k} \varphi^{k} ; \sum_{i=p}^{n} u_{i} \varphi^{i}\right)\right\}
$$

bei der Normierung

$$
\sum_{i=p}^{n} u_{i}^{2}=1
$$

Wir beachten, dass es hier und im folgenden genügt, wenn die betr. Abschätzungen für genügend grosse Zeiger $p$ d.h. für $p \geqq p_{0}\left(p_{0}\right.$ irgend eine und dann feste natürliche Zahl) erbracht werden, da sich daraus die Abschätzungen für die endlich vielen Zeiger $1 \leqq p<p_{0}$ von selbst ergeben.

Da im jetzigen Fall

$$
u=v=\sum_{i=p}^{n} u_{i} \varphi^{i} \quad \text { und } \quad\|u\|^{2}=\|v\|^{2}=\sum_{i=p}^{n} u_{i}^{2}=1
$$

ist, so folgt aus (21) eine Abschätzung

$$
\left|j_{1}^{*}(u ; u)\right| \leqq C[2 \sqrt{D[u]}+1] .
$$

Beachten wir noch die Beziehungen (27a) in $§ 6$ unter 3) und legen passend als Energieintegral den Ausdruck

$$
\{u, u\}=\sum_{i=p}^{n} x_{i} u_{i}^{2}
$$

zugrunde, so ergibt sich für das $j_{1}^{*}$ in (55) rechts eine Abschätzung 
(56)

$$
\left|j_{1}^{*}\left(\sum_{k=p}^{n} u_{k} \varphi^{k} ; \sum_{i=p}^{n} u_{i} \varphi^{i}\right)\right| \leqq A \sqrt{\sum_{i=p}^{n} \varkappa_{i} u_{i}^{2}}+B
$$

wobei $A>0, B>0$ zwei absolute Konstante sind. Wir verfügen über den festen Zeiger $p_{0}$ von oben so, dass $\varkappa_{p_{0}}>0$ und $A / \sqrt{\varkappa_{p_{0}}}<1$, sowie im Hinblick auf eine spätere Abschätzung, dass auch

$$
\max \left(\left|x_{1}\right|, \ldots,\left|x_{p}\right|\right)=\varkappa_{p} \text { für } p \geqq p_{0}
$$

ist. Mit der Abkürzung

$$
S=\sum_{i=p}^{n} x_{i} u_{i}^{2}
$$

ist der Ausdruck in den geschweiften Klammern in (55) rechts dann grösser gleich

$$
S-[A \sqrt{\bar{S}}+B]=S\left\{1-\frac{A}{\sqrt{S}}\right\}-B
$$

es ist aber (beachte $\sum_{i=p}^{n} u_{i}^{2}=1$ ) $S \geqq \varkappa_{p}$ und $1-A / \sqrt{S} \geqq 1-A / \sqrt{\varkappa_{p}}$ und a fortiori gilt dann

$$
\lambda_{p}^{n} \geqq \varkappa_{p}\left[1-\frac{A}{\sqrt{\varkappa_{p}}}\right]-B \text { für } p \geqq p_{0},
$$

wo die rechte Seite nicht von $n$ abhängt.

16. Die min-max-Eigenschaft von $\lambda_{p}^{n}$ besagt: wir bestimmen für $n-p$ Bindungen $\left(B_{\mu}\right)$ das Maximum der rechten Seite von (54) bei der Normierung

$$
\|u\|^{2}+\|v\|^{2}=\sum_{k=1}^{n} u_{k}^{2}+\sum_{i=1}^{n} v_{i}^{2}=2 ;
$$

alsdann ist $\lambda_{p}^{n}$ das Minimum dieser Maxima für alle solche möglichen Bindungen $\left(B_{\mu}\right)(\mu=1, \ldots, n-p)$. Wir wählen als solche Bindungen

$$
\left(B_{\mu}\right): \quad u_{p+1}=0, u_{p+2}=0, \ldots, u_{n}=0
$$

und erhalten

$$
\lambda_{p}^{n} \leqq \max \left\{\sum_{i=1}^{p} \varkappa_{i} u_{i} v_{i}+j_{1}^{*}\left(\sum_{k=1}^{p} u_{k} \varphi^{k} ; \sum_{i=1}^{n} v_{i} \varphi^{i}\right)\right\}
$$

bei der Normierung

$$
\sum_{k=1}^{p} u_{k}^{2}+\sum_{i=1}^{n} v_{i}^{2}=2
$$


Es ist bei dieser Normierung

$$
\sum_{i=1}^{p} \varkappa_{i} u_{i} v_{i} \leqq \varkappa_{p}
$$

für den Ausdruck $j_{1}^{*}$ gilt sinngemäss wieder die Abschätzung (56), wobei wir ohne Einschränkung $A$ und $B$ als dieselben Konstanten nehmen können, was

$$
\left|j_{1}^{*}\left(\sum_{k=1}^{p} u_{k} \varphi^{k} ; \sum_{i=1}^{n} v_{i} \varphi^{i}\right)\right| \leqq A \sqrt{\sum_{k=1}^{p} x_{k} u_{k}^{2}}+B
$$

liefert; da aber jetzt

$$
\sum_{k=1}^{p} \varkappa_{k} u_{k}^{2} \leqq 2 \varkappa_{p}
$$

ist, so folgt die zu (57) ganz analoge Abschätzung

$$
\lambda_{p}^{n} \leqq \varkappa_{p}\left[1+\frac{\sqrt{2} A}{\sqrt{\varkappa_{p}}}\right]+B \text { für } p \geqq p_{0},
$$

wo die rechte Seite wieder von $n$ nicht abhängt.

Ersetzen wir schliesslich $A$ und $B$ durch neue passende Konstante, die wir wieder mit $A, B$ bezeichnen, so erreichen wir, dass beide $\mathrm{Ab}$ schätzungen für alle $p \geqq 1$ gelten.

Eine Folgerung, die später gebraucht wird, ist diese: Sei $c>0$ beliebig und fest; dann existiert wegen (57) ein Zeiger $q$, so dass

$$
\lambda_{v}^{n} \geqq c>0
$$

für alle $v$ mit $q \leqq v \leqq n$ gilt. Alsdann zeigen (57) und (37), dass eine Abschätzung

$$
\sum_{\nu=q}^{n}\left(\lambda_{\nu}^{n}\right)^{-2} \leqq \Gamma^{*}
$$

gilt, wo $\Gamma^{*}>0$ eine von $n$ unabhängige Konstante ist.

17. Jetzt können wir für die zu $\lambda_{p}^{n}$ ( $p$ fest, $n$ wachsend) gehörige Folge $\left(U_{p}^{n}, V_{p}^{n}\right)$ einsehen, dass sie energiebeschränkt ist: $\left(U_{p}^{n}, V_{p}^{n}\right) \in E$. Beweis (vergl. auch [3], Seite 19): Für die $\zeta^{n}, \eta^{n}$ in (47) gelten die strengen Variationsgleichungen (48), wobei $\left.|| U_{p}^{n}\right|^{2}=\left\|V_{p}^{n}\right\|^{2}=1$ ist. Wir nehmen darin die erlaubten Ersetzungen vor, angedeutet durch das Zeichen $/ /$ :

$$
\eta^{n} / / V_{p}^{n} \quad \text { bzw. } \quad \eta^{n} / / U_{p}^{n} \quad \text { und } \quad \zeta^{n} / / V_{p}^{n}
$$

und erhalten

$$
I\left(U_{p}^{n} ; V_{p}^{n}\right)=\lambda_{p}^{n}
$$


bzw.

$$
I\left(U_{p}^{n} ; U_{p}^{n}\right)=I\left(V_{p}^{n} ; V_{p}^{n}\right)=\lambda_{p}^{n} H\left[U_{p}^{u}, V_{p}^{n}\right] .
$$

Wir setzen im weiteren Verlauf

$$
W_{p}^{n}=V_{p}^{n}-U_{p}^{n}
$$

und benutzen als Energieintegral passend den Ausdruck $I(f ; f)$. Es ist dann

$$
\lambda_{p}^{n}=I\left(U_{p}^{n} ; U_{p}^{n}\right)+I\left(U_{p}^{n} ; W_{p}^{n}\right) .
$$

Die Ersetzung $\eta^{n} / / W_{p}^{n}$ in (48) bringt

$$
I\left(U_{p}^{n} ; W_{p}^{n}\right)=\lambda_{p}^{n} H\left[V_{p}^{n}, W_{p}^{n}\right]=\lambda_{p}^{n}\left\{1-H\left[V_{p}^{n}, U_{p}^{n}\right]\right\}
$$

(beachte $\left.\left\|V_{p}^{n}\right\|^{2}=1, \ldots\right)$, wo $\quad 1-H\left[V_{p}^{n}, U_{p}^{n}\right] \geqq 0 \quad$ ist. Da also $I\left(U_{p}^{n} ; W_{p}^{n}\right) \geqq 0$ ist, so folgt aus (63) dass $\lambda_{p}^{n} \geqq I\left(U_{p}^{n} ; U_{p}^{n}\right)$ ist. Beachten wir noch (61), sowie (58), so kommt die in $n$ wieder gleichgradige Abschätzung

$$
I\left(U_{p}^{n} ; U_{p}^{n}\right)=I\left(V_{p}^{n} ; V_{p}^{n}\right) \leqq \varkappa_{p}\left[1+\frac{\sqrt{2} A}{\sqrt{\varkappa_{p}}}\right]+B,
$$

aus der wegen der Normierung der $U_{p}^{n}$ und $V_{p}^{n}$ folgt, dass $\left(U_{p}^{n}, V_{p}^{n}\right) \in E$ ist.

18. Aus dem vorigen Resultat ergibt sich die wichtige Tatsache: die Folge $\lambda_{p}^{n}$ ( $p$ fest, $n$ wachsend) hat nur endlich viele Häufungswerte.

Der Beweis ist wörtlich derselbe wie in [3], Seite 20-23, und braucht hier nicht wiederholt zu werden.

\section{Beweis, dass die Folge $\lambda_{p}^{n}$ konvergiert}

19. Im Gegensatz zu [3] ist hier der Beweis für die behauptete Konvergenz etwas schwieriger, da noch ein Randintegral auftritt, dessen Einfluss wir abschätzen müssen. Wir halten uns an die in $\S 11$ verabredete Sprech- und Schreibweise und legen in dem $2 n$-dimensionalen Raum $R^{2 n}$ die orthonormierte (so zu verstehen, dass jeder Vektor das Normquadrat 2 hat) Basis der Eigenvektoren zugrunde: wir setzen die Eigenvektoren und die dazu gehörigen Koordinaten, welche wir hier $x_{v}$ und $x_{v}^{\prime}$ nennen, wie folgt zusammen

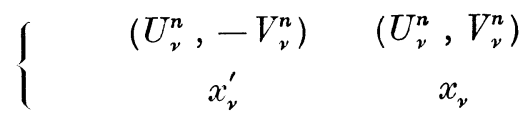

$(v=1, \ldots, n)$. Ist $(u, v)$ ein beliebiger $» V$ ektor» $\in R^{2 n}$, so gilt 


$$
(u, v)=\sum_{\nu=1}^{n} x_{\nu}^{\prime}\left(U_{\nu}^{n},-V_{\nu}^{n}\right)+\sum_{\nu=1}^{n} x_{\nu}\left(U_{\nu}^{n}, V_{\nu}^{n}\right)
$$

und es ist

$$
\|u\|^{2}+\|v\|^{2}=2 \sum_{\nu=1}^{n}\left(x_{\nu}^{\prime}\right)^{2}+2 \sum_{\nu=1}^{n}\left(x_{\nu}\right)^{2},
$$

da jeder Eigenvektor das Normquadrat 2 hat; die Normierung $\|u\|^{2}+$ $\|v\|^{2}=2$ drückt sich dann in den Koordinaten $x_{v}^{\prime}, x_{v}$ durch

$$
\sum_{v=1}^{n}\left(x_{\nu}^{\prime}\right)^{2}+\sum_{\nu=1}^{n}\left(x_{v}\right)^{2}=1
$$

aus. Wegen (49) erscheint $I(u ; v)$ in der Diagonalform

$$
I(u ; v)=-\sum_{\nu=1}^{n} \lambda_{\nu}^{n}\left(x_{\nu}^{\prime}\right)^{2}+\sum_{\nu=1}^{n} \lambda_{\nu}^{n}\left(x_{\nu}\right)^{2} .
$$

Und die Nebenbedingungen, die $\hat{\lambda}_{p}^{n}$ als grösstes Minimum liefern, lauten (67) $x_{n}^{\prime}=0, x_{n-1}^{\prime}=0, \ldots, x_{1}^{\prime}=0, x_{1}=0, \ldots, x_{p-1}=0$.

20. Von jetzt ab arbeiten wir in dem nächst höheren $\operatorname{Raum} R^{2 n+2}$, indem wir zu $\varphi^{1}, \ldots, \varphi^{n}$ noch die nächste Funktion $\varphi^{n+1}$ hinzunehmen. In diesem Raum $R^{2 n+2}$ erscheint der frühere Raum $R^{2 n}$ als ein Unterraum, den wir durch Hinzunahme der speziellen Vektoren

$$
\left\{\begin{array}{cc}
\left(\varphi^{n+1},-\varphi^{n+1}\right) & \left(\varphi^{n+1}, \varphi^{n+1}\right) \\
u_{n+1}^{\prime} & u_{n+1}
\end{array}\right.
$$

mit den zugehörigen Koordinaten $u_{n+1}^{\prime}$ bzw. $u_{n+1}$ ebenfalls auf die volle Dimension $2 n+2$ bringen. Wir beachten, dass die durch (65) und (68) gegebene Basis für den $R^{2 n+2}$ wieder orthonormiert ist (im obigen Sinne). Nehmen wir also in der Sprache dieser Basis zu den Bedingungen (67) noch die Bedingung $u_{n+1}^{\prime}=0$ hinzu, so ist nach der max-min-Eigenschaft von $\lambda_{p}^{n+1}$

$$
\lambda_{p}^{n+1} \geqq \min I(u ; v)=\varrho_{p}^{n+1},
$$

wobei

$$
(u, v)=\sum_{i=p}^{n} x_{i}\left(U_{i}^{n}, V_{i}^{n}\right)+u_{n+1}\left(\varphi^{n+1}, \varphi^{n+1}\right)
$$

und

$$
1=\sum_{i=p}^{n}\left(x_{i}\right)^{2}+\left(u_{n+1}\right)^{2}
$$


ist. Da für $u_{n+1}=0$ das $\min I(u ; v)=\lambda_{p}^{n}$ ist, so ist

$$
\varrho_{p}^{n+1} \leqq \lambda_{p}^{n} \text {. }
$$

Wir beachten die nach (35) gültigen Beziehungen $\left\{U_{k}^{n}, \varphi^{n+1}\right\}=0$, sowie $\left\{\varphi^{n+1}, V_{i}^{n}\right\}=0$, ferner $\left\{\varphi^{n+1}, \varphi^{n+1}\right\}=\varkappa_{n+1}$ und erinnern an die Relation (19), nach welcher z. B.

$$
I\left(U_{k}^{n} ; \varphi^{n+1}\right)=\left\{U_{k}^{n}, \varphi^{n+1}\right\}+j_{1}^{*}\left(U_{k}^{n} ; \varphi^{n+1}\right)
$$

ist, wo der erste Summand rechts verschwindet. Führen wir dann noch die Abkürzung

$$
A_{n+1}=\varkappa_{n+1}+j_{1}^{*}\left(\varphi^{n+1} ; \varphi^{n+1}\right)
$$

ein, so wird für das $(u, v)$ in $(70)$

$$
\begin{aligned}
& I(u ; v) \\
& =\sum_{i=p}^{n} \lambda_{i}^{n}\left(x_{i}\right)^{2}+\Lambda_{n+1}\left(u_{n+1}\right)^{2}+\sum_{k=p}^{n} x_{k} u_{n+1}\left[j_{1}^{*}\left(U_{k}^{n} ; \varphi^{n+1}\right)+j_{1}^{*}\left(\varphi^{n+1} ; V_{k}^{n}\right)\right] .
\end{aligned}
$$

Die eckige Klammer rechts formen wir wie folgt um: es ist nach (15) (beachte $j(u ; v)=-j(v ; u))$

$j_{1}^{*}\left(U_{k}^{n} ; \varphi^{n+1}\right)=j\left(\varphi^{n+1} ;-U_{k}^{n}\right)-\frac{1}{2} \int(\sigma-\tau) U_{k}^{n} \varphi^{n+1}$,

$j_{1}^{*}\left(\varphi^{n+1} ; V_{k}^{n}\right)=j\left(\varphi^{n+1} ; V_{k}^{n}\right)+\frac{1}{2} \int(\sigma-\tau) V_{k}^{n} \varphi^{n+1}-\left\{\int(\sigma-\tau) V_{k}^{n} \varphi^{n+1}\right\} ;$

addiert man spaltenweise, so kommt mit der Abkürzung (62) für den fraglichen Ausdruck

$$
\left\{j\left(\varphi^{n+1} ; W_{k}^{n}\right)+\frac{1}{2} \int(\sigma-\tau) \varphi^{n+1} W_{k}^{n}\right\}-\int(\sigma-\tau) \varphi^{n+1} V_{k}^{n},
$$

und hier ist die Klammer nach $(16)$ gerade $j_{2}^{*}\left(\varphi^{n+1} ; W_{k}^{n}\right)$, wodurch die Ableitungen auf $W_{k}^{n}$ verlagert sind, was für das Folgende wichtig ist. Wir setzen

$$
j_{2}^{*}\left(\varphi^{n+1} ; W_{k}^{n}\right)=2 a_{k}^{n}
$$

und bekommen für $I(u ; v)$ den endgültigen Ausdruck

$$
\begin{aligned}
& I(u ; v) \\
& =\left[\sum_{i=p}^{n} \lambda_{i}^{n}\left(x_{i}\right)^{2}+\Lambda_{n+1}\left(u_{n+1}\right)^{2}+2 \sum_{k=p}^{n} a_{k}^{n} x_{k} u_{n+1}\right] \\
& +\left\{\int(\sigma-\tau) u_{n+1} \varphi^{n+1}\left(\sum_{k=p}^{n} x_{k} V_{k}^{n}\right)\right\} .
\end{aligned}
$$


Und wir bemerken noch, dass nach (21) ( $n$ sei genügend gross)

$$
\left|j_{1}^{*}\left(\varphi^{n+1} ; \varphi^{n+1}\right)\right| \leqq C\left[2 \sqrt{D\left[\varphi^{n+1}\right]}+1\right]
$$

gilt; wegen $D\left[\varphi^{n+1}\right]=O\left(\left\{\varphi^{n+1}, \varphi^{n+1}\right\}\right)=O\left(\varkappa_{n+1}\right)$ gilt dann für das oben eingeführte $\Lambda_{n+1}$ nach (37) auch

$$
\Lambda_{n+1} \cong \frac{4 \pi(n+1)}{F}
$$

für $n \rightarrow \infty$.

21. Wir betrachten jetzt die Minimumslage der Form $I(u ; v)$ in (72) genauer. Bis auf Weiteres denken wir uns für die $x_{1}, \ldots, x_{n}, u_{n+1}$ die (bzw. solche) Werte genommen, für welche $I(u ; v)=\min I(u ; v)=\varrho_{p}^{n+1}$ ist; natürlich hängen diese $x_{i}$ und $u_{n+1}$ noch von $n$ ab, was wir aber in der Schreibung nicht zum Ausdruck bringen.

Für das Weitere ist es nun wichtig, dass die folgenden Abschätzungen gelten, in denen $W_{k}^{n}=V_{k}^{n}-U_{k}^{n}$ ist:

$$
\begin{aligned}
D\left[W_{k}^{n}\right] & =O(1), \\
\lambda_{k}^{n} H\left[W_{k}^{n}\right] & =O(1) ;
\end{aligned}
$$

dabei bedeutet das $O(1)$ jeweils eine absolute Konstante $>0$, die von $n$ und $k$ unabhängig ist $(1 \leqq k \leqq n)$. Der Beweis hierfür setzt nicht die Konvergenz der Folgen $\lambda_{p}^{n}$ voraus, und wird, um den jetzigen Gedankengang nicht zu unterbrechen, nachgetragen.

Trivial ist die folgende Abschätzung für die Energie von $u$ und $v$, wobei wir als Energieausdruck passend $\{f, f\}$ verwenden:

$$
\{u, u\} \leqq \varkappa_{n+1}, \quad\{v, v\} \leqq \varkappa_{n+1} .
$$

Ist nämlich z. B.

$$
v=\sum_{i=p}^{n} x_{i} V_{i}^{n}+u_{n+1} q^{n+1}=\sum_{v=1}^{n+1} \xi_{\nu} \varphi^{v}
$$

so ist

$$
\sum_{i=p}^{n} x_{i}^{2}+u_{n+1}^{2}=\sum_{\nu=1}^{n+1} \xi_{v}^{2}=1 \quad \text { und } \quad\{v, v\}=\sum_{\nu=1}^{n+1} x_{\nu} \xi_{v}^{2} \leqq \varkappa_{n+1},
$$

wie behauptet. Mithilfe davon erhalten wir eine erste Abschätzung des Randintegrales in $(72)$ von $I(u ; v)$. Es ist

$$
\left|\int(\sigma-\tau) u_{n+1} \varphi^{n+1}\left(\sum_{i=p}^{n} x_{i} V_{i}^{n}\right)\right| \leqq C\left|u_{n+1}\right| \int\left|\varphi^{n+1}\right|\left|\sum_{i=p}^{n} x_{i} V_{i}^{n}\right| .
$$


Das Integral wird nach der Standardabschätzung behandelt. Beachten wir die Normierung, so kommt

$$
\begin{aligned}
\int\left(\varphi^{n+1}\right)^{2} & =O\left(x_{n+1}^{1 / 2}\right), \\
\int\left(\sum_{i=p}^{n} x_{i} V_{i}^{n}\right)^{2} & =O\left(x_{n+1}^{1 / 2}\right),
\end{aligned}
$$

Letzteres, weil $\{v, v\} \leqq \varkappa_{n+1}$ ist, und daher

$$
\int(\sigma-\tau) u_{n+1} \phi^{n+1}\left(\sum_{i=p}^{n} x_{i} V_{i}^{n}\right)=O\left(x_{n+1}^{1 / 2}\left|u_{n+1}\right|\right) .
$$

Analog schätzen wir die Summe

$$
\sum_{k=p}^{n} a_{k}^{n} x_{k} u_{n+1}
$$

in (72) ab. Wenden wir auf $j_{2}^{*}\left(\varphi^{n+1} ; W_{k}^{n}\right)$ in (71) die Abschätzung (22) an und berücksichtigen (75), so ergibt sich

$$
\left|a_{k}^{n}\right| \leqq \alpha,
$$

wobei $\alpha>0$ eine von $k$ und $n$ unabhängige Konstante ist. Wir erhalten

$$
\left|\sum_{k=p}^{n} a_{k}^{n} x_{k} u_{n+1}\right| \leqq \alpha\left|u_{n+1}\right| \sum_{k=p}^{n}\left|x_{k}\right| ;
$$

die letzte Summe ist

$$
\leqq\left\{\sum_{k=p}^{n} x_{k}^{2} \sum_{k=p}^{n} 1^{2}\right\}^{1 / 2} \leqq \sqrt{n},
$$

und (beachte (37)) daher

$$
\| 2 \sum_{k=p}^{n} a_{k}^{n} x_{k} u_{n+1} \mid=O\left(x_{n+1}^{1 / 2}\left|u_{n+1}\right|\right) \text {. }
$$

Aus (77) und (79) folgt für unser $I(u ; v)$ in (72):

$$
\lambda_{p}^{n+1} \geqq \sum_{i=p}^{n} \lambda_{i}^{n}\left(x_{i}\right)^{2}+\Lambda_{n+1}\left(u_{n+1}\right)^{2}-O\left(x_{n+1}^{1 / 2}\left|u_{n+1}\right|\right),
$$

wobei $\Lambda_{n+1} \cong \varkappa_{n+1}$ ist. Daraus folgt, dass für die Minimumslage unmöglich $\lim \sup \left\{\Lambda_{n+1} u_{n+1}^{2}\right\}=+\infty$ für $n \rightarrow \infty$ sein kann; mit anderen Worten: es ist

$$
\Lambda_{n+1} u_{n+1}^{2}=O(1),
$$

und als Folge davon dann auch 


$$
\sum_{i=p}^{n} \lambda_{i}^{n} x_{i}^{2}=O(1)
$$

ferner gilt für das Minimum $\varrho_{p}^{n+1}$, dass auch $\left|\varrho_{p}^{n+1}\right|=O(1)$ ist.

22. Jetzt können wir die Energie von $u$ und $v$ besser abschätzen; wegen (80) genügt es, die Energie von $U$ und $V$, wo

$$
U=\sum_{i=p}^{n} x_{i} U_{i}^{n}, \quad V=\sum_{i=p}^{n} x_{i} V_{i}^{n}
$$

ist, abzuschätzen; wir setzen noch

$$
W=V-U=\sum_{i=p}^{n} x_{i} W_{i}^{n}
$$

Es ist wegen (81)

$$
I(U ; V)=\sum_{i=p}^{n} \lambda_{i}^{n}\left(x_{i}\right)^{2}=O(1)
$$

Aus

$$
\begin{aligned}
& I(U ; V)=I(V ; V)-I(W ; V) \\
& I(U ; V)=I(U ; U)+I(U ; W)
\end{aligned}
$$

folgt durch Addition

$$
2 I(U ; V)=I(U ; U)+I(V ; V)+\{I(U ; W)-I(W ; V)\} .
$$

Den Ausdruck in der geschweiften Klammer behandeln wir wie folgt weiter. Es ist

$$
I(U ; W)=\sum_{i=p}^{n} x_{i} I\left(U_{i}^{n} ; W\right) ;
$$

beachten wir, dass wir in den strengen Variationsgleichungen (48) die Ersetzung $\eta^{n} / / W$ machen dürfen, so ergibt sich

$$
I\left(U_{i}^{n} ; W\right)=\lambda_{i}^{n} H\left[V_{i}^{n}, W\right]
$$

und, wenn man für $W$ seinen Ausdruck einsetzt,

$$
I(U ; W)=\sum_{i=p}^{n} \sum_{k=p}^{n} x_{i} x_{k} \lambda_{i}^{n} H\left[V_{i}^{n}, W_{k}^{n}\right]
$$

Analog hat man die Gleichungen

und

$$
I(W ; V)=\sum_{i=p}^{n} x_{i} I\left(W ; V_{i}^{n}\right)==\sum_{i=p}^{n} x_{i} \lambda_{i}^{n} H\left[W, U_{i}^{n}\right]
$$

$$
I(W ; V)=\sum_{i=p}^{n} \sum_{k=p}^{n} x_{i} x_{k} \lambda_{i}^{n} H\left[U_{i}^{n}, W_{k}^{n}\right]
$$


Aus (84) und (85) folgt durch Subtraktion wegen $V_{i}^{n}-U_{i}^{n}=W_{i}^{n}$ :

$$
I(U ; W)-I(W ; V)=\sum_{i=p}^{n} \sum_{k=p}^{n} x_{i} x_{k} \lambda_{i}^{n} H\left[W_{i}^{n}, W_{k}^{n}\right] .
$$

Nun ist

$$
\left|x_{i} x_{k} \lambda_{i}^{n} H\left[W_{i}^{n}, W_{k}^{n}\right]\right| \leqq\left|x_{i}\right|\left|x_{k}\right| \lambda_{i}^{n}\left\|W_{i}^{n}\right\| \| W_{k}^{n} \mid
$$

und daher $|I(U ; W)-I(W ; V)|$ kleiner gleich

$$
\left\{\sum_{i=p}^{n} \sqrt{\lambda_{i}^{n}}\left|x_{i}\right|\left(\sqrt{\lambda_{i}^{n}}\left\|W_{i}^{n}\right\|\right)\right\} \sum_{k=p}^{n}\left|x_{k}\right|\left\|W_{k}^{n}\right\| .
$$

Wir schätzen diese Summen einzeln wie folgt ab. Die erste Summe ist wegen (75) ein

$$
O\left(\sum_{i=p}^{n} \sqrt{\lambda_{i}^{n}}\left|x_{i}\right|\right)
$$

also wegen

$$
\sum_{i=p}^{n} \sqrt{\lambda_{i}^{n}}\left|x_{i}\right| \leqq\left\{\sum_{i=p}^{n} \lambda_{i}^{n}\left(x_{i}\right)^{2} \sum_{i=p}^{n} 1^{2}\right\}^{1 / 2}
$$

und $(81)$ ein $O\left(x_{n+1}^{1 / 2}\right)$.

Die zweite Summe behandeln wir so: Ist $q$ der in (59) festgelegte Zeiger, so spalten wir diese in zwei Teilsummen:

$$
\sum_{k=p}^{q-1}\left|x_{k}\right|\left\|W_{k}^{n}\right\|+\sum_{v=q}^{n}\left|x_{v}\right|\left\|W_{v}^{n}\right\|
$$

(falls $q \leqq p$ ist, ist die erste Teilsumme leer, also gleich Null und die zweite gleich der alten Summe) auf, und bringen in der zweiten Teilsumme das allgemeine Glied auf die Gestalt

$$
\left\{\left(\lambda_{v}^{n}\right)^{-1 / 2}\left|x_{\nu}\right|\right\}\left\{\left(\lambda_{v}^{n}\right)^{1 / 2}\left\|W_{v}^{n}\right\|\right\} .
$$

Wegen (75) ist dann diese Teilsumme ein

$$
O\left(\sum_{\nu=q}^{n}\left(\lambda_{\nu}^{n}\right)^{-1 / 2}\left|x_{\nu}\right|\right)
$$

das allgemeine Glied dieser Summe schreiben wir in der Form

$$
\left\{\left(\lambda_{\nu}^{n}\right)^{1 / 2}\left|x_{\nu}\right|\right\}\left(\lambda_{\nu}^{n}\right)^{-1},
$$

schätzen die Summe nach der Schwarzschen Ungleichung ab und erhalten als obere Schranke

$$
\left\{\sum_{\nu=q}^{n} \lambda_{\nu}^{n}\left(x_{\nu}\right)^{2}\right\}^{1 / 2}\left\{\sum_{\nu=q}^{n}\left(\lambda_{\nu}^{n}\right)^{-2}\right\}^{1 / 2} ;
$$


diese Schranke ist aber nach (82) und (59) ein $O(1)$, und folglich auch der ganze Ausdruck (86) ein $O(1)$. Wir erhalten damit

$$
I(U ; W)-I(W ; V)=O\left(x_{n+1}^{1 / 2}\right) .
$$

Tragen wir diese Abschätzung in (83) rechts ein und beachten noch (82), so folgt

$$
I(U ; U)+I(V ; V)=O\left(x_{n+1}^{1 / 2}\right)
$$

und speziell

$$
D[V]=O\left(x_{n+1}^{1 / 2}\right)
$$

wo

$$
V=\sum_{i=p}^{n} x_{i} V_{i}^{n}
$$

ist.

23. Mithilfe der Abschätzung (87) folgt jetzt an Stelle von (76) die bessere Abschätzung

$$
\int\left(\sum_{i=p}^{n} x_{i} V_{i}^{n}\right)^{2}=O\left(x_{n+1}^{1 / 4}\right)
$$

Dadurch ergibt sich an Stelle der Abschätzung (77) für das Randintegral ebenfalls eine bessere: nämlich ein $O\left(\left(x_{n+1}^{1 / 2} x_{n+1}^{1 / 4}\right)^{1 / 2}\left|u_{n+1}\right|\right)$. Wir erhalten

$$
\int(\sigma-\tau) u_{n+1} \phi^{n+1}\left(\sum_{i=p}^{n} x_{i} V_{i}^{n}\right)=O\left(\varkappa_{n+1}^{3 / s}\left|u_{n+1}\right|\right) ;
$$

beachten wir jetzt noch (80) und bedenken, dass $A_{n+1} \cong \varkappa_{n+1}$ ist, so erhellt, dass die rechte Seite ein $O\left(\varkappa_{n+1}^{-1 / 8}\right)$ und also ein $o(1)$ ist. Wir notieren

$$
\int(\sigma-\tau) u_{n+1} \phi^{n+1}\left(\sum_{i=p}^{n} x_{i} V_{i}^{n}\right)=\varepsilon_{n}^{*},
$$

wo $\lim \varepsilon_{n}^{*}=0$ für $n \rightarrow \infty$ ist. Damit haben wir für die Minimumslage für unser $I(u ; v)$ in $(72)$

$$
\varrho_{p}^{n+1}=\left[\sum_{i=p}^{n} \lambda_{i}^{n}\left(x_{i}\right)^{2}+\Lambda_{n+1}\left(u_{n+1}\right)^{2}+2 \sum_{k=p}^{n} a_{k}^{n} x_{k} u_{n+1}\right]+\varepsilon_{n}^{*} .
$$

24. In (89) sind die $x_{i}$ und $u_{n+1}$ die festen Werte, für welche das Minimum $\varrho_{p}^{n+1}$ angenommen wird. Wir denken uns jetzt in der eckigen Klammer die $x_{p}, \ldots, x_{n}, u_{n+1}$ wieder variabel, behalten aber den Term $\varepsilon_{n}^{*}$ gemäss (88) bei und erhalten aus (89) die neue Abschätzung 


$$
\varrho_{p}^{n+1} \geqq \min \left[\sum_{i=p}^{n} \lambda_{i}^{n} x_{i}^{2}+\Lambda_{n+1} u_{n+1}^{2}+2 \sum_{k=p}^{n} a_{k}^{n} x_{k} u_{n+1}\right]-\left|\varepsilon_{n}^{*}\right|,
$$

wobei für das Minimum

$$
\sum_{i=p}^{n} x_{i}^{2}+u_{n+1}^{2}=1
$$

ist. Dieses neue Minimum sei $\sigma_{p}^{n+1}$, so dass also

$$
\varrho_{p}^{n+1} \geqq \sigma_{p}^{n+1}-\left|\varepsilon_{n}^{*}\right|
$$

ist. Da für $u_{n+1}=0$ das Minimum der Form in (90) gleich $\lambda_{p}^{n}$ ist, so folgt wieder

$$
\sigma_{p}^{n+1} \leqq \lambda_{p}^{n}
$$

Jetzt behaupten wir

$$
\lim \left\{\lambda_{p}^{n}-\sigma_{p}^{n+1}\right\}=0
$$

für $n \rightarrow \infty$.

Beweis indirekt (und gegenüber [3] vereinfacht). Wir nehmen also an, es existiere eine Teilfolge für $n$ mit der Eigenschaft, dass für sie

$$
\lambda_{p}^{n}-\sigma_{p}^{n+1} \geqq h>0
$$

ist, wo $h$ fest und unabhängig von $n$ ist. Stets durchlaufe im folgenden der Zeiger $n$ die genannte Teilfolge. Das Minimum $\sigma_{p}^{n+1}$ ist die kleinste Wurzel der Säkulargleichung, welche durch die folgende Gleichung in Determinantenform mit $\sigma$ als Variabler gegeben ist:

$$
\left|\begin{array}{ccccc}
\left(\lambda_{p}^{n}-\sigma\right) & \cdot & \cdot & \cdot & a_{p}^{n} \\
\cdot & \left(\lambda_{p+1}^{n}-\sigma\right) & \cdot & \cdot & a_{p+1}^{n} \\
\cdot & \cdot & \cdot & \cdot & \cdot \\
\cdot & \cdot & \cdot & \left(\lambda_{n}^{n}-\sigma\right) & a_{n}^{n} \\
a_{p}^{n} & a_{p+1}^{n} & . & a_{n}^{n} & \left(\Lambda_{n+1}-\sigma\right)
\end{array}\right|=0
$$

wobei alle übrigen Plätze mit Nullen besetzt sind. Denken wir uns darin $\sigma=\sigma_{p}^{n+1}$ eingetragen, so ist die Determinante Null und es existiert eine nichttriviale Lösung des zugehörigen homogenen Gleichungssystems

$$
\xi_{p}: \xi_{p+1}: \cdots: \xi_{n}: \xi_{n+1}
$$

wobei wir darauf verzichten, noch die Abhängigkeit von $n$ mitauszudrücken. Wegen (94) ist $\xi_{n+1} \neq 0$ und es kann daher $\xi_{n+1}=-1$ genommen werden. Es ergibt sich für diese Lösung 


$$
\begin{gathered}
\left(\lambda_{i}^{n}-\sigma_{p}^{n+1}\right) \xi_{i}=a_{i}^{n} \quad(i=p, \ldots, n) \\
\sum_{k=p}^{n} a_{k}^{n} \xi_{k}=\Lambda_{n+1}-\sigma_{p}^{n+1} .
\end{gathered}
$$

Wir berechnen die $\xi_{i}$ aus den ersten Gleichungen, tragen sie in die letzte Gleichung ein, und bekommen

$$
\Lambda_{n+1}-\sigma_{p}^{n+1}=\sum_{k=p}^{n} \frac{\left(a_{k}^{n}\right)^{2}}{\lambda_{k}^{n}-\sigma_{p}^{n+1}} .
$$

Hier ist die rechte Seite wegen (78), (94), (57) und (37) ein $O(\log n)$. Diese Aussage steht aber im Widerspruch zur linken Seite, welche (beachte (92) und (73)) bis auf den Faktor $4 \pi / F$ wie $n$ wächst.

25. Die damit bewiesene Behauptung (93) können wir auch so ausdrücken: es ist

$$
\sigma_{p}^{n+1}>\lambda_{p}^{n}-\tilde{\varepsilon}_{n},
$$

wo $\tilde{\varepsilon}_{n}>0$ und $\lim \tilde{\varepsilon}_{n}=0$ für $n \rightarrow \infty$ ist. Da nach $(69) \quad \lambda_{p}^{n+1} \geqq \varrho_{p}^{n+1}$ und nach (91) $\varrho_{p}^{n+1} \geqq \sigma_{p}^{n+1}-\left|\varepsilon_{n}^{*}\right|$ ist, so folgt mit $\varepsilon_{n}=\tilde{\varepsilon}_{n}^{\prime}+\left|\varepsilon_{n}^{*}\right|$ aus (95) eine Beziehung

$$
\lambda_{p}^{n+1}>\lambda_{p}^{n}-\varepsilon_{n},
$$

wobei $\varepsilon_{n}>0$ und $\lim \varepsilon_{n}=0$ für $n \rightarrow \infty$ ist. Diese Eigenschaft der Folge $\lambda_{p}^{n}$ zieht aber bekanntlich [4] nach sich, dass die Folge $\lambda_{p}^{n}$ zwischen ihrem unteren und oberen Häufungswert überall dicht liegt. Da sie nach $\S 18$ nur endlich viele Häufungswerte besitzt, so folgt notwendig, dass sie konvergiert:

$$
\lim \lambda_{p}^{n}=\lambda_{p}
$$

für $n \rightarrow \infty$ ( $p$ fest, $p=1,2, \ldots)$.

Aus (57) und (58) ergibt sich jetzt für diese Zahlen $\lambda_{p}$ die Abschätzung

$$
\varkappa_{p}\left[1-\frac{A}{\sqrt{\varkappa_{p}}}\right]-B \leqq \lambda_{p} \leqq \varkappa_{p}\left[1+\sqrt{2} \frac{A}{\sqrt{\varkappa_{p}}}\right]+B .
$$

Ferner ist

$$
0 \leqq \lambda_{1} \leqq \lambda_{2} \leqq \lambda_{3} \leqq \cdots
$$

und $\lim \lambda_{p}=\infty$ für $p \rightarrow \infty$. (96) zeigt, dass jedes $\lambda_{p}$ nur endlich oft auftritt. 


\section{Abschätzungen}

26. In den folgenden Abschätzungen schreiben wir den beliebigen Eigenvektor in der Form $\left(U_{p}^{n}, V_{p}^{n}\right)$, wo $1 \leqq p \leqq n$ ist. Es ist

$$
\frac{1}{2}\left\|W_{p}^{n}\right\|^{2}=\frac{1}{2}\left\|V_{p}^{n}-U_{p}^{n}\right\|^{2}=1-H\left[U_{p}^{n}, V_{p}^{n}\right] .
$$

Wir erinnern jetzt an die in $\S 17$ gewonnenen Ergebnisse und ersehen aus (64) und (98), dass

$$
I\left(U_{p}^{n} ; W_{p}^{n}\right)=\frac{1}{2} \lambda_{p}^{n}\left\|W_{p}^{n}\right\|^{2}
$$

ist. Ersetzen wir in (60) $V_{p}^{n}$ durch $U_{p}^{n}+W_{p}^{n}$ bzw. $U_{p}^{n}$ durch $V_{p}^{n}-W_{p}^{n}$, so erhalten wir

$$
\begin{aligned}
& \lambda_{p}^{n}=I\left(U_{p}^{n} ; U_{p}^{n}\right)+I\left(U_{p}^{n} ; W_{p}^{n}\right), \\
& \lambda_{p}^{n}=I\left(U_{p}^{n} ; U_{p}^{n}\right)-I\left(W_{p}^{n} ; V_{p}^{n}\right) .
\end{aligned}
$$

Die erste Beziehung ergibt nach (99)

$$
\lambda_{p}^{n}=I\left(U_{p}^{n} ; U_{p}^{n}\right)+\frac{1}{2} \lambda_{p}^{n}\left\|W_{p}^{n}\right\|^{2} .
$$

In (100) ersetzen wir im zweiten Glied abermals $U_{p}^{n}$ durch $V_{p}^{n}-W_{p}^{n}$ und bekommen

$$
\lambda_{p}^{n}=I\left(U_{p}^{n} ; U_{p}^{n}\right)+I\left(V_{p}^{n} ; W_{p}^{n}\right)-I\left(W_{p}^{n} ; W_{p}^{n}\right) .
$$

Addition von (101) und (103) gibt wegen $I(u ; v)-I(v ; u)=2 j(u ; v)$, wenn man noch durch 2 dividiert

$$
\lambda_{p}^{n}=I\left(U_{p}^{n} ; U_{p}^{n}\right)-\frac{1}{2} I\left(W_{p}^{n} ; W_{p}^{n}\right)+j\left(V_{p}^{n} ; W_{p}^{n}\right) .
$$

Da $j(u ; v)=-j(v ; u)$ ist, so gilt

$$
j\left(U_{p}^{n} ; W_{p}^{n}\right)=j\left(V_{p}^{n} ; W_{p}^{n}\right)=j\left(U_{p}^{n} ; V_{p}^{n}\right),
$$

und es wird aus (104)

$$
\lambda_{p}^{n}=I\left(U_{p}^{n} ; U_{p}^{n}\right)-\frac{1}{2} I\left(W_{p}^{n} ; W_{p}^{n}\right)+j\left(U_{p}^{n} ; V_{p}^{n}\right) .
$$

Für $j\left(U_{p}^{n} ; V_{p}^{n}\right)$ gilt nach (23) eine Abschätzung

$$
\left.\left|j\left(U_{p}^{n} ; V_{p}^{n}\right)\right| \leqq C\left[\sqrt{D\left[U_{p}^{n}\right.}\right]+\sqrt{D\left[V_{p}^{n}\right]}\right] .
$$


Nach (26) gilt weiter eine Abschätzung

$$
I\left(W_{p}^{n} ; W_{p}^{n}\right) \geqq-2 K,
$$

wo $K>0$ eine Konstante ist, die nicht von $p$ und $n$ abhängt. Berücksichtigen wir jetzt noch (27a) sowie $I\left(U_{p}^{n} ; U_{p}^{n}\right)=I\left(V_{p}^{n} ; V_{p}^{n}\right)$ (vergleiche (61)), so folgt aus (106), (107) und (108) die Aussage

$$
\lambda_{p}^{n} \leqq I\left(U_{p}^{n} ; U_{p}^{n}\right)+K+O\left(\sqrt{I\left(U_{p}^{n} ; U_{p}^{n}\right)}\right) ;
$$

aus ihr ergibt sich

$$
\lim \inf \left\{\left(\lambda_{p}^{n}\right)^{-1} I\left(U_{p}^{n} ; U_{p}^{n}\right)\right\} \geqq 1 \quad(p \rightarrow \infty)
$$

"gleichgradig" für alle $n$ mit $n \geqq p$.

Anderseits ist, im selben Sinne verstanden, wegen $\lambda_{p}^{n} \geqq I\left(U_{p}^{n} ; U_{p}^{n}\right)$ (man vergleiche (102))

$$
\lim \sup \left\{\left(\lambda_{p}^{n}\right)^{-1} I\left(U_{p}^{n} ; U_{p}^{n}\right)\right\} \leqq 1 \quad(p \rightarrow \infty) .
$$

Beide Aussagen zusammen zeigen, dass der folgende Limes für $p \rightarrow \infty$ gleichgradig in $n$ existiert und gleich 1 ist:

$$
\lim \left\{\left(\lambda_{p}^{n}\right)^{-1} I\left(U_{p}^{n} ; U_{p}^{n}\right)\right\}=1 .
$$

Wegen $I\left(U_{p}^{n} ; U_{p}^{n}\right)=I\left(V_{p}^{n} ; V_{p}^{n}\right)$ gilt dieselbe Beziehung auch für $V_{p}^{n}$ anstelle von $U_{p}^{n}$.

27. Wir knüpfen an (106) an und formen das Glied $j\left(U_{p}^{n} ; V_{p}^{n}\right)$ um. Nach (105) ist es gleich $j\left(U_{p}^{n} ; W_{p}^{n}\right)$. (15) ergibt (beachte $j(u ; v)=$ $\left.j_{1}(u ; v)\right)$

$$
j\left(U_{p}^{n} ; V_{p}^{n}\right)=j_{1}^{*}\left(U_{p}^{n} ; W_{p}^{n}\right)+\frac{1}{2} \int(\sigma-\tau) U_{p}^{n} W_{p}^{n}
$$

Da die linke Seite nach (105) auch gleich $j\left(V_{p}^{n} ; W_{p}^{n}\right)$ ist, so gilt nach (16) auch

$$
j\left(U_{p}^{n} ; V_{p}^{n}\right)=j_{2}^{*}\left(V_{p}^{n} ; W_{p}^{n}\right)-\frac{1}{2} \int(\sigma-\tau) V_{p}^{n} W_{p}^{n} .
$$

Wir addieren diese Beziehungen, berücksichtigen im Randbeitrag $V_{p}^{n}-U_{p}^{n}=$ $W_{p}^{n}$, dividieren noch durch 2 und erhalten

$$
j\left(U_{p}^{n} ; V_{p}^{n}\right)=\frac{1}{2} j_{\mathrm{I}}^{*}\left(U_{p}^{n} ; W_{p}^{n}\right)+\frac{1}{2} j_{2}^{*}\left(V_{p}^{n} ; W_{p}^{n}\right)-\frac{1}{4} \int(\sigma-\tau)\left(W_{p}^{n}\right)^{2} .
$$

Wir tragen diesen Ausdruck in (106) ein, fassen passend zusammen, und bekommen mit der Abkürzung 
(109)

$$
A_{p}^{n}=I\left(W_{p}^{n} ; W_{p}^{n}\right)-j_{2}^{*}\left(V_{p}^{n} ; W_{p}^{n}\right)+\frac{1}{2} \int(\sigma-\tau)\left(W_{p}^{n}\right)^{2}
$$

die Beziehung

$$
\lambda_{p}^{n}-I\left(U_{p}^{n} ; U_{p}^{n}\right)=\frac{1}{2} j_{1}^{*}\left(U_{p}^{n} ; W_{p}^{n}\right)-\frac{1}{2} A_{p}^{n},
$$

wo die linke Seite nach (102) gleich $\frac{1}{2} \lambda_{p}^{n}\left\|W_{p}^{n}\right\|^{2}$ ist. Wir beachten, dass in $j_{2}^{*}\left(V_{p}^{n} ; W_{p}^{n}\right)$ die Ableitungen auf $W_{p}^{n}$ verlagert sind und daher wegen (22)

$$
j_{2}^{*}\left(V_{p}^{n} ; W_{p}^{n}\right)=O\left(\sqrt{D\left[W_{p}^{n}\right]}\right)=O\left(\sqrt{\left|I\left(W_{p}^{n} ; W_{p}^{n}\right)\right|}\right)
$$

ist; dieselbe Aussage gilt nach der Courantschen Ungleichung für das Randintegral in (109). Das heisst aber, dass in dem Ausdruck $A_{p}^{n}$ das Integral $I\left(W_{p}^{n} ; W_{p}^{n}\right)$ führt, und daher

$$
A_{p}^{n} \geqq-L
$$

ist, wo $L>0$ eine von $n$ und $p$ unabhängige Konstante ist. So folgt aus (110), wo die linke Seite gleich $\frac{1}{2} \lambda_{p}^{n}\left\|W_{p}^{n}\right\|^{2}$ ist:

$$
\lambda_{p}^{n}\left\|W_{p}^{n}\right\|^{2} \leqq j_{1}^{*}\left(U_{p}^{n} ; W_{p}^{n}\right)+L
$$

ist.

Das Glied $j_{1}^{*}\left(U_{p}^{n} ; W_{p}^{n}\right)$ schätzen wir nach (21) ab:

$$
\left|j_{1}^{*}\left(U_{p}^{n} ; W_{p}^{n}\right)\right| \leqq C\left\{2 \sqrt{D\left[U_{p}^{n}\right]}+\sqrt{H\left[U_{p}^{n}\right]}\right\} \sqrt{H\left[W_{p}^{n}\right]} .
$$

Da $D\left[U_{p}^{n}\right]$ ein $O\left(I\left(U_{p}^{n} ; U_{p}^{n}\right)\right)$ ist und $I\left(U_{p}^{n} ; U_{p}^{n}\right) \leqq \lambda_{p}^{n}$ ist, so folgt

$$
\left|j_{1}^{*}\left(U_{p}^{n} ; W_{p}^{n}\right)\right| \leqq M \sqrt{\lambda_{p}^{n}\left\|W_{p}^{n}\right\|^{2}},
$$

wo $M>0$ eine von $n$ und $p$ unabhängige Konstante ist. Aus (111) und (112) folgt jetzt

$$
\lambda_{p}^{n}\left\|W_{p}^{n}\right\|^{2}=O(1),
$$

wo das $O(1)$ eine von $n$ und $p$ unabhängige Konstante ausdrückt.

Mit dieser Erkenntnis kehren wir zu der Beziehung (110) zurück und schreiben sie so:

$$
A_{p}^{n}=j_{1}^{*}\left(U_{p}^{n} ; W_{p}^{n}\right)-\lambda_{p}^{n}\left\|W_{p}^{n}\right\|^{2} \leqq j_{1}^{*}\left(U_{p}^{n} ; W_{p}^{n}\right) .
$$

Nach (112) und (113) ist $j_{1}^{*}\left(U_{p}^{n} ; W_{p}^{n}\right)$ ein $O(1)$, also in (114) das $A_{p}^{n}$ ein $O(1)$ und daher auch das Führungsintegral $I\left(W_{p}^{n} ; W_{p}^{n}\right)$ in $A_{p}^{n}$ ein $O(1)$, und mithin

$$
D\left[W_{p}^{n}\right]=O(1),
$$

wo das $O(1)$ eine von $n$ und $p$ unabhängige Konstante ausdrückt. 


\section{Die Existenz von Eigenfolgen}

28. Wir erinnern an das in $\S 11$ Gebrachte und wissen, dass in dem dort mit $R^{2 n}$ bezeichneten Raum die strengen Variationsgleichungen (48) gelten. Im folgenden ist $p$ ein beliebiger fester Zeiger, während $n(n \geqq p)$ wächst. Nach § 17 wissen wir, dass die »Funktionen» $\left(U_{p}^{n}, V_{p}^{n}\right)$ energiebeschränkt sind:

$$
\left(U_{p}^{n}, V_{p}^{n}\right) \in E .
$$

Es sei jetzt $\left(f^{n}, g^{n}\right)$ eine beliebige Folge von Funktionen, welche ebenfalls energiebeschränkt sind, so dass also

$$
\left(f^{n}, g^{n}\right) \in E
$$

ist. Wir ersetzen in den strengen Variationsgleichungen $\left(\zeta^{n}, \eta^{n}\right)$ durch $\left(f^{n}, g^{n}\right)$ und zeigen, dass dann diese Gleichungen noch in dem Sinne gelten, dass in den Gleichungen (48) rechts ein $o(1)$ anstelle der Null steht:

$$
\begin{aligned}
& I\left(U_{p}^{n} ; g^{n}\right)-\lambda_{p}^{n} H\left[V_{p}^{n}, g^{n}\right]=o(1), \\
& I\left(f^{n} ; V_{p}^{n}\right)-\lambda_{p}^{n} H\left[f^{n}, U_{p}^{n}\right]=o(1) .
\end{aligned}
$$

Wir beweisen z. B. die zweite Beziehung (115b). Dazu entwicklen wir $f^{n}$ in bezug auf das System $\varphi^{\nu}$

$$
f^{n} \sim \sum_{v=1}^{\infty} f_{v}^{n} \varphi^{v}
$$

setzen

$$
\zeta^{n}=\sum_{\nu=1}^{n} f_{v}^{n} \varphi^{\nu} \quad \text { und } \quad F^{n}=f^{n}-\zeta^{n},
$$

wo also $F^{n}$ orthogonal zu $\varphi^{1}, \varphi^{2}, \ldots, \varphi^{n}$ ist. Wir schreiben die beiden folgenden Gleichungen an, multiplizieren sie mit den rechts angeschriebenen Faktoren 1 und $-\hat{\lambda}_{p}^{n}$ und addieren anschliessend:

$$
\begin{array}{rl|l}
I\left(f^{n} ; V_{p}^{n}\right) & =I\left(\zeta^{n} ; V_{p}^{n}\right)+I\left(F^{n} ; V_{p}^{n}\right), & 1 \\
H\left[f^{n}, U_{p}^{n}\right] & =H\left[\zeta^{n}, U_{p}^{n}\right]+H\left[F^{n}, U_{p}^{n}\right] . & -\lambda_{p}^{n}
\end{array}
$$

Die erste Spalte rechts liefert Null nach (48); ferner ist $H\left[F^{n}, U_{p}^{n}\right]=o(1)$. Weiter ist nach (19)

$$
I\left(F^{n} ; V_{p}^{n}\right)=\left\{F^{n}, V_{p}^{n}\right\}+j_{i}^{*}\left(F^{n} ; V_{p}^{n}\right),
$$

und hier verschwindet nach (35) der erste Summand rechts. Weiter ist nach (15) und (16)

$$
j_{1}^{*}\left(F^{n} ; V_{p}^{n}\right)=j_{2}^{*}\left(F^{n} ; V_{p}^{n}\right)-\int(\sigma-\tau) F^{n} V_{p}^{n} .
$$


Da $\quad V_{p}^{n}$ energiebeschränkt und $H\left[F^{n}\right]=o(1)$ ist, so ist nach der $\mathrm{Ab}$ schätzung (22) auch $j_{2}^{*}\left(F^{n} ; V_{p}^{n}\right)=o(1)$. Für das Randintegral gilt aus denselben Gründen auf Grund der Courantschen Gleichung, dass es ein $o(1)$ ist. Damit ist (115b) bewiesen. Analog beweist man die Aussage (115a).

Nutzen wir jetzt noch die Konvergenz von $\lambda_{p}^{n}$ aus und tragen in (115a) und (115b) $\lambda_{p}^{n}=\lambda_{p}+o(1)$ ein, so gelten diese Aussagen unverändert mit $\lambda_{p}$ anstelle von $\lambda_{p}^{n}$. Schliesslich schreiben wir $\left(\zeta^{n}, \eta^{n}\right)$ für $\left(f^{n}, g^{n}\right)$ und sehen, dass für eine beliebige Folge $\left(\zeta^{n}, \eta^{n}\right)$ mit

$$
\left(\zeta^{n}, \eta^{n}\right) \in E
$$

die Variationsgleichungen

$$
\left\{\begin{array}{l}
I\left(U_{p}^{n} ; \eta^{n}\right)-\lambda_{p} H\left[V_{p}^{n}, \eta^{n}\right]=o(1), \\
I\left(\zeta^{n} ; V_{p}^{n}\right)-\lambda_{p} H\left[\zeta^{n}, U_{p}^{n}\right]=o(1)
\end{array}\right.
$$

gelten. Nach der in $\S 12$ gegebenen Definition heisst das aber: $\lambda_{p}$ ist Eigenwert und $\left(U_{p}^{n}, V_{p}^{n}\right)$ eine zugehörige Eigenfolge unseres Variationsproblems $I(u ; v)=$ stationär für alle Funktionen $(u, v) \in \Omega$ mit $\|u\|^{2}+\|v\|^{2}=2$.

\section{Die Vollständigkeit des Systems der Funktionenfolgen $\left\{U_{p}^{n}\right\}$}

29. Im Gegensatz zu [3] bringt es der bezüglich der Randbedingungen unsymmetrische $\varphi$ - $\varphi$-Ansatz mit sich, dass wir hier nur die Vollständigkeit der ersten Folge $\left\{U_{p}^{n}\right\}$ von $\left(U_{p}^{n}, V_{p}^{n}\right)$ beweisen können. Wir beachten im folgenden, dass die Funktionen $U_{p}^{n}, V_{p}^{n}$ in dem von $\varphi^{1}, \ldots, \varphi^{n}$ aufgespannten linearen Teilraum liegen, und dass dort die Funktionen $U_{1}^{n}, \ldots, U_{n}^{n}$ und $V_{1}^{n}, \ldots, V_{n}^{n}$ je einen Satz von $n$ orthonormierten Funktionen bilden $($ Norm $=1)$.

Definition: Es sei $f$ eine stückweise stetige Funktion mit $H[f]<\infty$. Es sei ferner $\varepsilon>0$ beliebig vorgegeben. Das System der Funktionen $\left\{U_{p}^{n}\right\}$ heisst vollständig, wenn es einen Zeiger $N$ und passende Zahlenfolgen $A_{1}^{n}, \ldots, A_{N}^{n}$ gibt derart, dass

$$
\lim \sup \left\{\left\|f-\left\{A_{1}^{n} U_{1}^{n}+\cdots+A_{N}^{n} U_{N}^{n}\right\}\right\|\right\} \leqq \varepsilon
$$

für $n \rightarrow \infty$ gilt.

Wir setzen im folgenden voraus, dass $\zeta^{n}, \eta^{n}$ dem von $\varphi^{1}, \ldots, \varphi^{n}$ aufgespannten Raum angehören. Dann gelten die strengen Variationsgleichungen (48). Indem wir $I(u ; v)$ noch gemäss (19) aufspalten, lauten diese Gleichungen

$$
\begin{aligned}
& \left\{U_{p}^{n}, \eta^{n}\right\}+j_{1}^{*}\left(U_{p}^{n} ; \eta^{n}\right)-\lambda_{p}^{n} H\left[V_{p}^{n}, \eta^{n}\right]=0, \\
& \left\{\zeta^{n}, V_{p}^{n}\right\}+j_{1}^{*}\left(\zeta^{n} ; V_{p}^{n}\right)-\lambda_{p}^{n} H\left[\zeta^{n}, U_{p}^{n}\right]=0 .
\end{aligned}
$$


A. I. 401

Ferner benötigen wir die Variationsgleichung (33) für die Funktionen $\varphi^{i}$; wir schreiben sie für $\varphi^{r}$ nochmals an:

$$
\left\{\varphi^{r}, \zeta\right\}-\varkappa_{r} H\left[\varphi^{r}, \zeta\right]=0 .
$$

Es sei $n$ fest und es seien die Zeiger $r$ und $m$ wie folgt beschränkt: $1 \leqq r \leqq m \leqq n$. Dann sind in (118) und (117b) die folgenden Ersetzungen erlaubt:

was die Aussagen

$$
\zeta / / V_{p}^{n}, \quad \zeta^{n} / / \varphi^{r},
$$

$$
\begin{aligned}
& 0=\left\{\phi^{r}, V_{p}^{n}\right\}-\varkappa_{r} H\left[\varphi^{r}, V_{p}^{n}\right], \\
& 0=\left\{\varphi^{r}, V_{p}^{n}\right\}+j_{1}^{*}\left(\varphi^{r} ; V_{p}^{n}\right)-\lambda_{p}^{n} H\left[\varphi^{r}, U_{p}^{n}\right]
\end{aligned}
$$

liefert, aus denen sich

$$
\lambda_{p}^{n} H\left[\psi^{r}, U_{p}^{n}\right]=\varkappa_{r} H\left[\psi^{r}, V_{p}^{n}\right]+j_{1}^{*}\left(\psi^{r} ; V_{p}^{n}\right)
$$

ergibt. Analog liefern die Ersetzungen $\zeta / / C_{p}^{n}$ und $\eta^{n} / / \varphi^{r}$ die zu (119) analoge Gleichung

$$
\lambda_{p}^{n} H\left[V_{p}^{n}, \varphi^{r}\right]=\varkappa_{r} H\left[\psi^{r}, U_{p}^{n}\right]+j_{1}^{*}\left(U_{p}^{n} ; \varphi^{r}\right) .
$$

Führen wir die »Fourierkoeffizienten» von $\varphi^{r}$ bezüglich $U_{1}^{n}, \ldots, U_{n}^{n}$ bzw. $V_{1}^{n}, \ldots, V_{n}^{n}$ gemäss

$$
a_{r p}^{n}=H\left[\varphi^{r}, U_{p}^{n}\right], \quad b_{r p}^{n}=H\left[\varphi^{r}, V_{p}^{n}\right]
$$

ein, so ist

$$
\varphi^{r}=\sum_{p=1}^{n} a_{r p}^{n} U_{p}^{n}=\sum_{p=1}^{n} b_{r_{p}}^{n} V_{p}^{n}
$$

mit

$$
\sum_{p=1}^{n}\left(a_{r p}^{n}\right)^{2}=\sum_{p=1}^{n}\left(b_{r_{p}}^{n}\right)^{2}=1 .
$$

Die Beziehungen (119) und (120) lauten dann

$$
\begin{aligned}
& \lambda_{p}^{n} a_{r p}^{n}=\varkappa_{r} b_{r p}^{n}+j_{1}^{*}\left(\phi^{r} ; T_{p}^{n}\right), \\
& \lambda_{p}^{n} b_{r p}^{n}=\varkappa_{r} a_{r p}^{n}+j_{1}^{*}\left(U_{p}^{n} ; \varphi^{r}\right) .
\end{aligned}
$$

Von diesen zwei Aussagen hilft uns nur die erste weiter, in welcher in $j_{1}^{*}\left(\varphi^{r} ; V_{p}^{n}\right)$ die Ableitungen von $V_{p}^{n}$ nicht vorkommen. Wir multiplizieren in (121a) mit $a_{r p}^{n}$ und summieren über $p$; mit der Abkürzung

$$
\hat{V}=\sum_{p=1}^{n} a_{r p}^{n} V_{p}^{n}, \quad H[\hat{V}]=1
$$


ergibt sich

$$
\sum_{p=1}^{n} \lambda_{p}^{n}\left(a_{r p}^{n}\right)^{2}=\varkappa_{r} \sum_{p=1}^{n} a_{r p}^{n} b_{r p}^{n}+j_{1}^{*}\left(\varphi^{r} ; \hat{V}\right) .
$$

Hier ist die Summe rechts absolut kleiner gleich $1 ; j_{1}^{*}$ nach (21) abgeschätzt, gibt wegen $H[\hat{V}]=1$

$$
\left|j_{1}^{*}\left(\varphi^{r} ; \hat{V}\right)\right| \leqq C\left[2 \sqrt{D\left[\varphi^{r}\right]}+1\right],
$$

und hierin ist $D\left[\varphi^{r}\right] \leqq \gamma\left|\varkappa_{r}\right|$, wo $\gamma>0$ eine absolute Konstante bedeutet. Mit der Abkürzung

$$
K_{r}=\left|\varkappa_{r}\right|+C\left[2 \sqrt{\gamma\left|\varkappa_{r}\right|}+1\right]
$$

ergibt sich jetzt aus (122) die Abschätzung

$$
\sum_{p=1}^{n} \lambda_{p}^{n}\left(a_{r p}^{n}\right)^{2} \leqq K_{r}
$$

30. Seien jetzt $f$ und $\varepsilon>0$ wie in der Definition gegeben und ohne Einschränkung $\|f\|>0$. Es sei

$$
f \sim \sum_{\nu=1}^{\infty} f_{\nu} \varphi^{\nu}, \quad\|f\|^{2}=\sum_{\nu=1}^{\infty} f_{\nu}^{2} ;
$$

hier haben wir ausgenutzt, dass die $\varphi^{\nu}$ ein vollständiges System bilden. Es existiert dann ein Zeiger $m=m(\varepsilon)$ so, dass

$$
\left\|f-\sum_{r=1}^{m} f_{r} \varphi^{r}\right\| \leqq \frac{\varepsilon}{2}
$$

ist. $m$ ist jetzt fest und $1 \leqq r \leqq m$. Es sei

$$
K=\max \left(K_{1}, K_{2}, \ldots, K_{m}\right) \text {. }
$$

Wir bestimmen im Hinblick auf (57) ein $N$ so, dass

$$
m K \leqq\left(\frac{\varepsilon}{2}\|f\|^{-1}\right)^{2}\left\{\varkappa_{N+1}\left[1-\frac{A}{\sqrt{\varkappa_{N+1}}}\right]-B\right\}
$$

wird. Ferner wählen wir aus einem Grund, der später klar wird, den Zeiger $N$ noch so, dass

$$
\lambda_{N}<\lambda_{N+1}
$$

ist. Für $1 \leqq r \leqq m$, dieses $N$ und für $n>N$ folgt aus (123) und wegen (57) 


$$
K \geqq \sum_{p=N+1}^{n} \lambda_{p}^{n}\left(a_{r p}^{n}\right)^{2} \geqq\left\{\varkappa_{N+1}\left[1-\frac{A}{\sqrt{\varkappa_{N+1}}}\right]-B\right\} \sum_{p=N+1}^{n}\left(a_{r p}^{n}\right)^{2} .
$$

Summiert man über $r(1 \leqq r \leqq m)$ und vergleicht mit (125) so kommt

$$
\sum_{r=1}^{m} \sum_{p=N+1}^{n}\left(a_{r p}^{n}\right)^{2} \leqq\left(\frac{\varepsilon}{2}\|f\|^{-1}\right)^{2} \text {. }
$$

Die links erfassten Zeiger $r$ und $p$ entstammen in dem nachstehenden Schema der Fourierkoeffizienten dem stark umrahmten Rechteck

\begin{tabular}{c|ccccc|ccccc|} 
& $U_{1}^{n}$ & $\cdot$ & $\cdot$ & $\cdot$ & $U_{N}^{n}$ & $U_{N+1}^{n}$ & $\cdot$ & $\cdot$ & $\cdot$ & $U_{n}^{n}$ \\
\hline$\varphi^{1}$ & $a_{11}^{n}$ & $\cdot$ & $\cdot$ & $\cdot$ & $a_{1 N}^{n}$ & $a_{1, N+1}^{n}$ & $\cdot$ & $\cdot$ & $\cdot$ & $a_{1 n}^{n}$ \\
$\varphi^{2}$ & $a_{21}^{n}$ & $\cdot$ & $\cdot$ & $\cdot$ & $a_{2 N}^{n}$ & $a_{2, N+1}^{n}$ & $\cdot$ & $\cdot$ & $\cdot$ & $a_{2 n}^{n}$ \\
$\cdot$ & $\cdot$ & $\cdot$ & $\cdot$ & $\cdot$ & $\cdot$ & $\cdot$ & $\cdot$ & $\cdot$ & $\cdot$ & $\cdot$ \\
$\cdot$ & $\cdot$ & $\cdot$ & $\cdot$ & $\cdot$ & $\cdot$ & $\cdot$ & $\cdot$ & $\cdot$ & $\cdot$ & $\cdot$ \\
$\varphi^{m}$ & $a_{m 1}^{n}$ & $\cdot$ & $\cdot$ & $\cdot$ & $a_{m N}^{n}$ & $a_{m, N+1}^{n}$ & $\cdot$ & $\cdot$ & $\cdot$ & $a_{m n}^{n}$ \\
\hline
\end{tabular}

Wir multiplizieren die Zeilen dieser Matrix der Reihe nach mit den Koeffizienten $f_{1}, \ldots, f_{m}$, die in (124) auftreten, addieren und erhalten mit der Abkürzung

die Beziehung

$$
A_{v}^{n}=\sum_{r=1}^{m} f_{r} a_{r v}^{n}
$$

$$
\sum_{r=1}^{m} f_{r} \varphi^{r}=\sum_{i=1}^{N} A_{i}^{n} U_{i}^{n}+\sum_{v=N_{+1}}^{n} A_{v}^{n} U_{v}^{n}
$$

Aus (127) folgt

$$
\left(A_{v}^{n}\right)^{2} \leqq \sum_{r=1}^{m} f_{r}^{2} \sum_{r=1}^{m}\left(a_{r v}^{n}\right)^{2} \leqq\|f\|^{2} \sum_{r=1}^{m}\left(a_{r v}^{n}\right)^{2}
$$

Das Normquadrat der zweiten Summe in (128) rechts schätzen wir nach oben ab durch $\left(A_{N+1}^{n}\right)^{2}+\cdots+\left(A_{n}^{n}\right)^{2}$, was wegen (129) gerade die linke Seite von (126), noch multipliziert mit $\|f\|^{2}$, liefert, das ist eine Zahl die $\leqq(\varepsilon / 2)^{2}$ ist. Daraus und aus (128) folgt für die Normen selbst

$$
\left\|\sum_{r=1}^{m} f_{r} \varphi^{r}-\sum_{i=1}^{N} A_{i}^{n} U_{i}^{n}\right\| \leqq \frac{\varepsilon}{2} .
$$

Zusammen mit (124) ergibt sich

$$
\left\|f-\left\{A_{1}^{n} U_{1}^{n}+\cdots+A_{N}^{n} U_{N}^{n}\right\}\right\| \leqq \varepsilon
$$


für alle $n>N$. Ersetzt man darin die Koeffizienten $A_{v}^{n}$ durch die Fourierkoeffizienten

$$
\alpha_{v}^{n}=H\left[f, U_{v}^{n}\right],
$$

so gilt, wie bekannt, erst recht

$$
\left\|f-\left\{\alpha_{1}^{n} U_{1}^{n}+\cdots+\alpha_{N}^{n} U_{N}^{n}\right\}\right\| \leqq \varepsilon
$$

für $n>N$ und es ist

$$
\left(\alpha_{1}^{n}\right)^{2}+\cdots+\left(\alpha_{N}^{n}\right)^{2} \leqq\|f\|^{2} .
$$

Die Beziehungen (130) bzw. (131) drücken aus, dass das System der Funktionen $\left\{U_{v}^{n}\right\}$ im Sinne der oben gegebenen Dəfinition vollständig ist.

31. Wir stellen uns jetzt in dem vorhergehenden Gedankengang vor, dass die Funktion $f$ Element aus einem Raum $E$ ist:

$$
E=\left\{f \mid H[f] \leqq \Gamma_{1}, D[f] \leqq \Gamma_{2}\right\} \text {. }
$$

Für die Fourierkoeffizienten $f_{v}$ gilt dann nach (42) eine "gleichgradige» Abschätzung

$$
\sum_{\nu=m+1}^{\infty}\left(f_{\nu}\right)^{2} \leqq M \varkappa_{m+1}^{-1},
$$

wo $M>0$ nur von dem Raum $E$, jedoch nicht von dem einzelnen $f$ abhängt. Wird also $m$ so gewählt, dass die rechte Seite $\leqq \varepsilon / 2$ wird, so gilt wieder die Aussage (124) gleichgradig für alle $f \in E$. Der weitere Gedankengang verläuft jetzt wie in $\S 30$ und führt zu den Beziehungen (131) und (132).

Durchläuft $f$ speziell eine Folge $f^{n}$, so gilt mit den Koeffizienten

$$
F_{v}^{n}=H\left[f^{n}, U_{v}^{n}\right]
$$

nach (131) und (132)

$$
\left\|f-\left\{F_{1}^{n} U_{1}^{n}+\cdots+F_{N}^{n} U_{N}^{n}\right\}\right\| \leqq \varepsilon
$$

für $n>N$ und es ist

$$
\left(F_{1}^{n}\right)^{2}+\cdots+\left(F_{N}^{n}\right)^{2} \leqq\left\|f^{n}\right\|^{2}=O(1) .
$$

32. Wir können jetzt zeigen:

Es gibt keine Eigenfolge $\left(U^{n}, V^{n}\right)$ zu einem Eigenwert $\Lambda \geqq 0$, die zu allen Eigenfolgen $\left(U_{p}^{n},-V_{p}^{n}\right)$ und $\left(U_{p}^{n}, V_{p}^{n}\right)$ orthogonal ist.

Beweis: Nach der Definition für eine Eigenfolge ist $\left(U^{n}, V^{n}\right) \in E$, also speziell $U^{n} \in E$, d.h. wir können auf die Folge $U^{n}$ das Ergebnis von $\S 31$ 
A. I. 401

anwenden. Ferner liegt in der Voraussetzung, dass $U^{n}$ bereits einzeln orthogonal zu allen $U_{p}^{n}$ ist, d.h. es ist

$$
\alpha_{v}^{n}=H\left[U^{n}, U_{v}^{n}\right]=o(1)
$$

für $n \rightarrow \infty$ und jedes feste $v$. Nach $\S 31$ gibt es zu vorgegebenen $\varepsilon>0$ einen Zeiger $N$, so dass (133) für $n>N$ gilt $\left(f^{n} \operatorname{durch} U^{n}\right.$ und $F_{v}^{n}$ durch $\alpha_{v}^{n}$ ersetzt). Der Limes $n \rightarrow \infty$ liefert dann wegen (135)

$$
\lim \sup \left\|U^{n}\right\| \leqq \varepsilon
$$

für $n \rightarrow \infty$, also, da $\varepsilon$ beliebig war,

$$
H\left[U^{n}\right]=o(1) .
$$

Nach $\S 12,2^{\circ}$ gilt aber für eine Eigenfolge $H\left[U^{n}\right]=1+o(1)$ im Widerspruch $\mathrm{zu}(136)$.

Aus dem damit bewiesenen Ergebnis folgt:

Es existiert keine Eigenfolge $\left(U^{n}, V^{n}\right)$, die zu einem Eigenwert $\Lambda \neq \lambda_{p}$ $(p=1,2, \ldots)$ gehört.

Denn nach dem in $\S 12,5^{\circ}$ Gesagten wäre diese Folge $\left(U^{n}, V^{n}\right)$ orthogonal zu allen Eigenfolgen $\left(U_{p}^{n},-V_{p}^{n}\right)$ und $\left(U_{p}^{n}, V_{p}^{n}\right)$. Mit anderen Worten:

Als mögliche Eigenwerte kommen nur die bereits festgestellten $\lambda_{p}(p=$ 1,2,..) in Frage.

33. Hier setzen wir voraus, dass $\left(U^{n}, V^{n}\right)$ eine verallgemeinerte Eigenfolg e zum Wert $\Lambda \geqq 0$ ist. Es gelten dann die Variationsgleichungen

$$
\begin{aligned}
& I\left(U^{n} ; \eta^{n}\right)-\Lambda H\left[V^{n}, \eta^{n}\right]=o(1), \\
& I\left(\zeta^{n} ; V^{n}\right)-\Lambda H\left[\zeta^{n}, U^{n}\right]=o(1) .
\end{aligned}
$$

W eiter setzen wir voraus, dass diese Folge $\left(U^{n}, V^{n}\right)$ zu allen Eigenfolgen $\left(U_{p}^{n},-V_{p}^{n}\right)$ und $\left(U_{p}^{n}, V_{p}^{n}\right)$ orthogonal ist.

Wir können dann den Gedankengang von $\S 32$ auf $\left(U^{n}, V^{n}\right)$ anwenden bis zu der Folgerung (136), die wir noch einmal anschreiben:

$$
H\left[U^{n}\right]=o(1) .
$$

Die Ersetzung $\eta^{n} / / U^{n}$ in (137a) liefert dann

$$
I\left(U^{n} ; U^{n}\right)-\Lambda H\left[V^{n}, U^{n}\right]=o(1),
$$

und hier ist $H\left[V^{n}, U^{n}\right]=o(1)$ nach (138), woraus

$$
I\left(U^{n} ; U^{n}\right)=o(1)
$$

folgt. Nach dem in $\S 6$ unter 5) Gesagten besagen (138) und (139) zusammen

$$
U^{n} \sim 0 \text {. }
$$


Mit dieser Erkenntnis kehren wir zu der Gleichung (137a) zurück und machen die Ersetzung $\eta^{n} / / V^{n}$ was

$$
I\left(U^{n} ; V^{n}\right)-\Lambda H\left[V^{n}\right]=o(1)
$$

liefert. Der erste Term links ist wegen (140) ein $o(1)$, so dass aus (141) folgt

$$
\Lambda H\left[V^{n}\right]=o(1) \text {. }
$$

Ferner liefert die Ersetzung $\zeta^{n} / / V^{n}$ in der Gleichung (137b)

$$
I\left(V^{n} ; V^{n}\right)-\Lambda H\left[V^{n}, U^{n}\right]=o(1),
$$

also wegen (140)

$$
I\left(V^{n} ; V^{n}\right)=o(1) .
$$

Ist $A>0$, so folgt aus (142) und (143) dass auch $V^{n} \sim 0$ ist. Wir notieren das Ergebnis:

Ist $\Lambda>0$, so ist $\left(U^{n}, V^{n}\right) \sim(0,0)$; ist $\Lambda=0$, so ist $U^{n} \sim 0$ und $I\left(V^{n} ; V^{n}\right)=o(1)$.

Bemerkung: In [3] erhielten wir auch im Falle $\Lambda=0$ die Aussage $\left(U^{n}, V^{n}\right) \sim(0,0)$. Der Grund für diesen Unterschied liegt darin, dass wir hier nur die Vollständigkeit des Systems $\left\{U_{v}^{n}\right\}$ beweisen konnten. Da wir aber auch im Falle $\Lambda=0$ die Aussage $\left(U^{n}, V^{n}\right) \sim(0,0)$ später brauchen, müssen wir nun den $\psi$ - $\psi$-Ansatz (9) heranziehen.

\section{Die entsprechenden Aussagen beim $\psi$ - $\psi$-Ansatz}

34. Wir erinnern uns jetzt daran, dass alle Betrachtungen, die wir bislang für den $\varphi$ - $\varphi$-Ansatz angestellt und alle Ergebnisse, die wir für ihn gewonnen haben, sinngemäss auch für den $\psi$ - $\psi$-Ansatz gelten. Wir erhalten so zu jeder früheren Aussage beim $\varphi$ - $\varphi$-Ansatz eine dazu »spiegelbildliche» beim $\psi$ - $\psi$-Ansatz. Wir brauchen das nicht auszuführen, sondern uns nur das »Lexikon» einzuprägen, welches die Übersetzung der beiden Ansätze ineinander leistet.

An die Stelle der Funktionen $\phi^{i}$ treten die Funktionen $\psi^{i}$, an die Stelle des Courantschen Problems I tritt jetzt das Problem II, und die $\mu_{p}$ beim Problem II übernehmen die Rolle der bisher benutzten $\varkappa_{p}$. Dementsprechend wird jetzt mit der Identität (20) anstelle von (19) gearbeitet. Es seien $\varrho_{p}^{n}$ und $\left(X_{p}^{n}, Y_{p}^{n}\right)$ die Grössen, welche an die Stelle der $\lambda_{p}^{n}$ und $\left(U_{p}^{n}, V_{p}^{n}\right)$ treten. Wieder gilt, dass $\lim \varrho_{p}^{n}=\varrho_{p}$ für $n \rightarrow \infty$ existiert, und dass $\left(X_{p}^{n}, Y_{p}^{n}\right)$ eine zu $\varrho_{p}$ gehörige Eigenfolge ist, wobei die Definition der Eigenfolge $\left(X^{n}, Y^{n}\right)$ für einen Eigenwert $\varrho$ dieselbe wie 
früher ist. Ebenso gelten die entsprechenden Abschätzungen: so tritt z. B. an die Stelle von (96) die Abschätzung

$$
\mu_{p}\left[1-\frac{A^{*}}{\sqrt{\mu_{p}}}\right]-B^{*} \leqq \varrho_{p} \leqq \mu_{p}\left[1+\sqrt{2} \frac{A^{*}}{\sqrt{\mu_{p}}}\right]+B^{*},
$$

wobei $A^{*}>0, B^{*}>0$ absolute Konstante sind.

35. Den in $\S 32$ und $\S 33$ festgestellten Tatsachen entsprechen hier die folgenden:

$1^{\circ}$. Das System der Funktionen $\left\{Y_{p}^{n}\right\}$ ist vollständig.

$2^{\circ}$. Es gibt keine Eigenfolge $\left(X^{n}, Y^{n}\right)$ zu einem Eigenwert $P \geqq 0$, die zu allen Eigenfolgen $\left(X_{p}^{n},-Y_{p}^{n}\right)$ und $\left(X_{p}^{n}, Y_{p}^{n}\right)$ orthogonal ist.

$3^{\circ}$. Es existiert keine Eigenfolge $\left(X^{n}, Y^{n}\right)$, die zu einem Eigenwert $P \neq \varrho_{p}(p=1,2, \ldots)$ gehört, mit anderen Worten:

$4^{\circ}$. Als mögliche Eigenwerte kommen nur die bereits festgestellten $\varrho_{p}$ $(p=1,2, \ldots)$ in Frage.

$5^{\text {o. }}$. Ist $\left(X^{n}, Y^{n}\right)$ eine verallgemeinerte Eigenfolge zum Eigenwert $P \geqq 0$, die orthogonal $\mathrm{zu}$ allen Eigenfolgen $\left(X_{p}^{n},-Y_{p}^{n}\right)$ und $\left(X_{p}^{n}, Y_{p}^{n}\right)$ ist, so gilt:

Ist $P>0$, so ist $\left(X^{n}, Y^{n}\right) \sim(0,0)$; ist $P=0$, so ist $Y^{n} \sim 0$ und $I\left(X^{n} ; X^{n}\right)=o(1)$.

\section{Abgeänderte Eigenfolgen}

36. Beim $\varphi$ - $\varphi$-Ansatz erfüllt nur $U_{p}^{n}$ die vorgeschriebene Randbedingung, jedoch nicht $V_{p}^{n}$; umgekehrt erfüllt beim $\psi$ - $\psi$-Ansatz nur $Y_{p}^{n}$ die vorgeschriebene Randbedingung, jedoch nicht $X_{p}^{n}$. Im Hinblick auf die $\mathrm{zu}$ erbringenden Existenzbeweise und auch aus allgemeinen Gründen wünscht man aber "möglichst kräftige» Eigenfolgen, wozu gehört, dass beide Partner die vorgeschriebenen Randbedingungen erfüllen.

Wir gehen aus von dem $\varphi$ - $\varphi$-Ansatz, projizieren $V_{p}^{n}$ auf den $\psi$-Raum, der von den $\psi^{1}, \psi^{2}, \ldots$ aufgespannt wird,

$$
V_{p}^{n} \sim \sum_{\nu=1}^{\infty} V_{p \nu}^{n} \psi^{\nu}
$$

und setzen

$$
\tilde{V}_{p}^{n}=\sum_{i=1}^{n} V_{p^{i}}^{n} \psi^{i} \text { und } \hat{V}_{p}^{n}=V_{p}^{n}-\tilde{V}_{p}^{n}
$$

Dann ist

$$
V_{p}^{n}=\tilde{V}_{p}^{n}+\hat{V}_{p}^{n},
$$


und es gehört $\tilde{V}_{p}^{n}$ dem von $\psi^{1}, \ldots, \psi^{n}$ aufgespannten Raum an, während $\hat{V}_{p}^{n}$ orthogonal zu $\psi^{1}, \ldots, \psi^{n}$ ist. Wir wissen, dass $V_{p}^{n}$ energiebeschränkt ist ( $p$ ist fest). Legen wir also für $V_{p}^{n}$ jetzt passend als Energieintegral den Ausdruck $[f, f]$ zugrunde, so folgt auf Grund der beim Problem II in (40b) festgestellten Eigenschaften der $\psi^{i}$ aus (144) die Beziehung

$$
\left[V_{p}^{n}, V_{p}^{n}\right]=\left[\tilde{V}_{p}^{n}, \tilde{V}_{p}^{n}\right]+\left[\hat{V}_{p}^{n}, \hat{V}_{p}^{n}\right]
$$

aus der hervorgeht, dass auch $\tilde{V}_{p}^{n}$ und $\hat{V}_{p}^{n}$ energiebeschränkt sind, und dass

$$
H\left[\hat{V}_{p}^{n}\right]=o(1)
$$

ist.

Da $\hat{V}_{p}^{n} \in E$ ist, so ist in den Variationsgleichungen (116) die Ersetzung $\zeta^{n} / / \hat{V}_{p}^{n}$ erlaubt, was

$$
I\left(\hat{V}_{p}^{n} ; V_{p}^{n}\right)=\lambda_{p} H\left[\hat{V}_{p}^{n}, U_{p}^{n}\right]+o(1)
$$

liefert. Hier ist der erste Summand rechts nach (145) ein $o(1)$. Anderseits ist die linke Seite nach (20)

$$
\left[\hat{V}_{p}^{n}, V_{p}^{n}\right]+j_{2}^{*}\left(\hat{V}_{p}^{n} ; V_{p}^{n}\right) ;
$$

da in $j_{2}^{*}\left(\hat{V}_{p}^{n} ; V_{p}^{n}\right)$ die Ableitungen von $\hat{V}_{p}^{n}$ nicht auftreten und $V_{p}^{n}$ energiebeschränkt ist, so ist wegen (145) auch $j_{2}^{*}\left(\hat{V}_{p}^{n} ; V_{p}^{n}\right)$ ein $o(1)$; da $\left[\hat{V}_{p}^{n}, V_{p}^{n}\right]=\left[\hat{V}_{p}^{n}, \hat{V}_{p}^{n}\right]$ ist, so folgt

$$
\left[\hat{V}_{p}^{n}, \hat{V}_{p}^{n}\right]=o(1)
$$

(145) und (147) besagen zusammen

$$
\hat{V}_{p}^{n} \sim 0 \quad \text { bzw. } \quad V_{p}^{n} \sim \tilde{V}_{p}^{n} .
$$

37. Jetzt projizieren wir auch noch $U_{p}^{n}$ auf den Raum der $\psi^{i}$. In der zu (144) analogen Bezeichnung

$$
U_{p}^{n}=\tilde{U}_{p}^{n}+\hat{U}_{p}^{n}
$$

ergibt sich wieder: auch $\tilde{U}_{p}^{n}$ und $\hat{U}_{p}^{n}$ sind energiebeschränkt und

$$
H\left[\hat{U}_{p}^{n}\right]=o(1) \text {. }
$$

Da $\hat{U}_{p}^{n} \in E$ ist, so ist in (116) die Ersetzung $\eta^{n} / / \hat{U}_{p}^{n}$ erlaubt und es ergibt sich

$$
I\left(U_{p}^{n} ; \hat{U}_{p}^{n}\right)=\lambda_{p} H\left[V_{p}^{n}, \hat{U}_{p}^{n}\right]+o(1)
$$


wo der erste Summand rechts nach (149) ein $o(1)$ ist. Die linke Seite ist analog zu (146) gleich

$$
\left[U_{p}^{n}, \hat{U}_{p}^{n}\right]+j_{2}^{*}\left(U_{p}^{n} ; \hat{U}_{p}^{n}\right)
$$

wo der erste Summand gleich $\left[\hat{U}_{p}^{n}, \hat{U}_{p}^{n}\right]$ ist. Gegenüber $\S 36$ müssen wir in dem zweiten Summanden die Ableitungen auf $U_{p}^{n}$ verlagern gemäss der Beziehung

$$
j_{2}^{*}\left(U_{p}^{n} ; \hat{U}_{p}^{n}\right)=j_{1}^{*}\left(U_{p}^{n} ; \hat{U}_{p}^{n}\right)+\int(\sigma-\tau) U_{p}^{n} \hat{U}_{p}^{n},
$$

und sehen, dass der erste Summand rechts wegen (149) ein o(1) ist. Der Randbeitrag ist es aber nach der Courantschen Ungleichung auch, da bei der Abschätzung als Faktor $\left\{H\left[\hat{U}_{p}^{n}\right]\right\}^{1 / 4}=o(1)$ auftritt. Somit ist auch jetzt

$$
\left[\hat{U}_{p}^{n}, \hat{U}_{p}^{n}\right]=o(1)
$$

daraus und aus (149) ergibt sich

$$
\hat{U}_{p}^{n} \sim 0 \quad \text { bzw. } \quad U_{p}^{n} \sim \tilde{U}_{p}^{n} .
$$

38. Dual zu den in $\S 36$ und $\S 37$ gewonnenen Resultaten ergibt sich, wenn wir von dem $\psi$ - $\psi$-Ansatz ausgehen, und $X_{p}^{n}, Y_{p}^{n}$ auf den Raum der $\varphi^{i}$ projizieren

$$
X_{p}^{n}=\tilde{X}_{p}^{n}+\hat{X}_{p}^{n}, \quad Y_{p}^{n}=\tilde{Y}_{p}^{n}+\hat{Y}_{p}^{n},
$$

wobei $\tilde{X}_{p}^{n}$ und $\tilde{Y}_{p}^{n}$ dem von den $\varphi^{1}, \ldots, \varphi^{n}$ aufgespannten Teilraum angehören, dass

$$
\left(\hat{X}_{p}^{n}, \hat{Y}_{p}^{n}\right) \sim(0,0)
$$

bzw. dass

$$
\left(X_{p}^{n}, Y_{p}^{n}\right) \sim\left(\tilde{X}_{p}^{n}, \tilde{Y}_{p}^{n}\right)
$$

ist.

\section{Folgerungen}

39. Wir beachten, dass die Variationsgleichungen (116) unempfindlich gegenüber einer Ersetzung $V_{p}^{n} / / \tilde{V}_{p}^{n}$ sind, wo $V_{p}^{n} \sim \tilde{V}_{p}^{n}$ ist. Dann ergibt sich aus $\S 36$, dass

$$
\left(U_{p}^{n}, \tilde{V}_{p}^{n}\right)
$$


eine Eigenfolge zum Wert $\lambda_{p}$ ist, bei welcher nun auch der zweite Partner $\tilde{V}_{p}^{n}$ die vorgeschriebene Randbedingung erfüllt. Dieselbe Aussage gilt für die Folge

$$
\left(\tilde{X}_{p}^{n}, Y_{p}^{n}\right)
$$

bei welcher jetzt auch der erste Partner $\tilde{X}_{p}^{n}$ die richtige Randbedingung erfüllt.

40. Aus demselben Grunde ergibt sich aus $\S 37$, dass die Folge

$$
\left(\tilde{U}_{p}^{n}, \tilde{V}_{p}^{n}\right)
$$

Eigenfolge zum Wert $\lambda_{p}$ beim $\psi$ - $\psi$-Ansatz ist. Und ebenso ist

$$
\left(\tilde{X_{p}^{n}}, \tilde{Y}_{p}^{n}\right)
$$

Eigenfolge zum Wert $\varrho_{p}$ beim $\varphi$ - $\varphi$-Ansatz.

Dieselben Tatsachen gelten auch für eine verallgemeinerte Eigenfolge: ist also z. B. $\left(U^{n}, V^{n}\right)$ eine verallgemeinerte Eigenfolge zum Wert $\Lambda \geqq 0$ beim $\varphi$ - $\varphi$-Ansatz, so ist $\left(\tilde{U}^{n}, \tilde{V}^{n}\right)$ eine verallgemeinerte Eigenfolge zum selben Wert $\Lambda$ beim $\psi$ - $\psi$-Ansatz, und umgekehrt.

Nach $\S 32$, Schluss und $\S 35,4^{\circ}$ ergibt sich: jedes $\lambda_{p}$ ist gleich einem $\varrho_{v}$ und jedes $\varrho_{p}$ gleich einem $\lambda_{\mu}$.

Nehmen wir weiter an, dass $\left(U^{n}, V^{n}\right)$ eine verallgemeinerte Eigenfolge zum Eigenwert 0 beim $\varphi$ - $\varphi$-Ansatz ist, welche $\mathrm{zu}$ allen Eigenfolgen $\left(U_{p}^{n},-V_{p}^{n}\right)$ und $\left(U_{p}^{n}, V_{p}^{n}\right)$ orthogonal ist, so ist nach $\S 33$, Schluss

$$
U^{n} \sim 0, \quad I\left(V^{n} ; V^{n}\right)=o(1) .
$$

Da aber $\left(\tilde{U}^{n}, \tilde{V}^{n}\right)$ auch eine verallgemeinerte Eigenfolge zum Wert 0 beim $\psi$ - $\psi$-Ansatz ist, welche ebenfalls zu allen Eigenfolgen $\left(X_{p}^{n},-Y_{p}^{n}\right)$ und $\left(X_{p}^{n}, Y_{p}^{n}\right)$ des $\psi$ - $\psi$-Ansatzes orthogonal ist, so gilt nach $\S 35$, Schluss, dass auch

$$
I\left(\tilde{U}^{n} ; \tilde{U}^{n}\right)=o(1), \quad \tilde{V}^{n} \sim 0
$$

ist. Beide Aussagen (152) und (153) ergeben

$$
\left(U^{n}, V^{n}\right) \sim(0,0) .
$$

Die entsprechende Tatsache gilt beim $\psi$ - $\psi$-Ansatz: ist $\left(X^{n}, Y^{n}\right)$ eine verallgemeinerte Eigenfolge zum Wert 0 des $\psi$ - $\psi$-Ansatzes, die zu allen Eigenfolgen $\left(X_{p}^{n},-Y_{p}^{n}\right)$ und $\left(X_{p}^{n}, Y_{p}^{n}\right)$ orthogonal ist, so ist

$$
\left(X^{n}, Y^{n}\right) \sim(0,0) .
$$

Dass die Aussagen (154) bzw. (155) für einen Eigenwert $\Lambda>0$ bzw. $P>0$ gelten, haben wir schon früher in $\S 33$ und $\S 35$ bewiesen. 
41. Mit dem zuletzt gewonnenen Ergebnis stehen wir aber jetzt unter denselben Voraussetzungen wie in [3], § 13. Es gilt daher wie dort für den $\varphi$ - $\varphi$-Ansatz:

Ist $\left(U^{n}, V^{n}\right)$ eine Eigenfolge, so ist sie unter den früher konstruierten Eigenfolgen $\left(U_{p}^{n}, V_{p}^{n}\right)$ enthalten.

Dieselbe Tatsache gilt für den $\psi$ - $\psi$-Ansatz.

Der Beweis verläuft wörtlich wie in [3].

Beachten wir den ersten Teil von $\S 40$, so folgt, dass notwendig

$$
\lambda_{p}=\varrho_{p}
$$

ist, und dass der $\varphi$ - $\varphi$-Ansatz äquivalent mit dem $\psi$ - $\psi$-Ansatz ist.

42. Wir können jetzt zeigen, dass auch das System der Funktionen $\left\{V_{p}^{n}\right\}$ vollständig ist. Dazu gehen wir von $\psi$ - $\psi$-Ansatz aus und stützen uns auf die Vollständigkeit des Systems $\left\{Y_{p}^{n}\right\}$. Für dieses System gelten die zu (131) und (132) analogen Aussagen

$$
\begin{gathered}
\left\|f-\left\{\gamma_{1}^{n} Y_{1}^{n}+\cdots+\gamma_{N}^{n} Y_{N}^{n}\right\}\right\| \leqq \varepsilon, \\
\left(\gamma_{1}^{n}\right)^{2}+\cdots+\left(\gamma_{N}^{n}\right)^{2} \leqq\|f\|^{2}
\end{gathered}
$$

für $n>N$. Ersetzen wir $Y_{v}^{n}$ durch $\tilde{Y}_{v}^{n}$, so bleibt wegen (157) die Aussage (156) gültig bis auf einen Fehler, der ein $o(1)$ ist ( $N$ ist fest!):

$$
\left\|f-\left\{\gamma_{1}^{n} \tilde{Y}_{1}^{n}+\cdots+\gamma_{N}^{n} \tilde{Y}_{N}^{n}\right\}\right\| \leqq \varepsilon+o(1) .
$$

Die zu diesen $N$ Funktionen $\tilde{Y}_{v}^{n}(v=1, \ldots, N)$ gehörigen Eigenfolgen $\left(\tilde{X}_{v}^{n}, \tilde{Y}_{v}^{n}\right)$ sind nach $\S 40$ aber Eigenfolgen des $\varphi$ - $\varphi$-Ansatzes und daher nach $\S 41$ unter den Eigenfolgen $\left(U_{p}^{n}, V_{p}^{n}\right)$ enthalten. Wegen $\lambda_{v}=o_{v}$ und weil in $\S 30$ der Zeiger $N$ so gewählt wurde, dass $\lambda_{N}<\lambda_{N+1}$ ist, kommen für den Zeiger $p$ nur die Zahlen $1, \ldots, N$ in Frage. Nach der in $\S 12$ unter $4^{\circ}$ gegebenen Definition der linearen Abhängigkeit von Eigenfolgen ergibt sich dann, dass bis auf einen Fehler, der wieder ein $o(1)$ ist, die (158) entsprechende Aussage

$$
\left\|f-\left\{\delta_{1}^{n} V_{1}^{n}+\cdots+\delta_{N}^{n} V_{N}^{n}\right\}\right\| \leqq \varepsilon+o(1)
$$

mit neuen Koeffizienten $\delta_{v}^{n}$ gilt. Umso mehr gilt dann mit den FourierKoeffizienten

dass

$$
\beta_{v}^{n}=H\left[f, V_{v}^{n}\right]
$$

$$
\begin{gathered}
\left\|f-\left\{\beta_{1}^{n} V_{1}^{n}+\cdots+\beta_{N}^{n} V_{N}^{n}\right\}\right\| \leqq \varepsilon+o(1), \\
\left(\beta_{1}^{n}\right)^{2}+\cdots+\left(\beta_{n}^{N}\right)^{2} \leqq\|f\|^{2}
\end{gathered}
$$

ist, das System $\left\{V_{p}^{n}\right\}$ also vollständig ist.

Analog erweist sich beim $\psi$ - $\psi$-Ansatz das System $\left\{X_{p}^{n}\right\}$ als vollständig. 


\section{Beweis der Lorentz-Sommerfeldschen Vermutung}

43. Nach $\S 39$ und $\S 41$ haben wir in den $\left(U_{p}^{n}, \tilde{V}_{p}^{n}\right)$ und $\lambda_{p}$ sämtliche Eigenfolgen und Eigenwerte des Variationsproblems

$$
I(u ; v)=\text { stationär bei }\|u\|^{2}+\|v\|^{2}=2 .
$$

Dabei erfüllt $U_{p}^{n}$ die Randbedingung (1a) und $\tilde{V}_{p}^{n}$ die Randbedingung (1b). Ferner ist nach (96) und (37) für $p \rightarrow \infty$

$$
\lambda_{p} \simeq \frac{4 \pi p}{F}
$$

( $F$ der Inhalt des Gebietes), d.h. es gilt die Lorentz-Sommerfeldsche Vermutung. Zum Beweis benötigten wir nicht die Existenz von Eigenfunktionen, sondern lediglich die viel leichter einsehbare Existenz von Eigenfolgen.

\section{Zusatz}

Herr Professor Dr. G. L. Tautz (Universität Freiburg im Breisgau) war so freundlich, auch diese Arbeit kritisch durchzusehen. In einem ausführlichen Brief vom 9. Oktober 1964, für den ich Herrn Tautz auch an dieser Stelle herzlich danke, bemerkt er unter anderem:

1) Der Existenzbeweis für die Eigenfunktionen steckt bereits in unseren Abschätzungen. Denn unsere Eigenfolgen sind ja energiebeschränkt, also normbeschränkt nach der Norm des Hilbertraums

$$
\|\varphi\|^{1}=\{H[\varphi]+D[\varphi]\}^{1 / 2}<\infty ;
$$

ihm entspricht in unserem Text der Raum $E$. Benutzt man den zugehörigen abgeschlossenen Raum, so kann man den bekannten Satz anwenden, dass man aus einer normbeschränkten Folge eine schwach konvergente auswählen kann, die also schwach gegen ein Element desselben Raumes strebt. Diese Funktionen erfüllen wegen der schwachen Konvergenz und wegen der Konvergenz der $\lambda_{p}^{n}$ die Variationsgleichungen, sind also "schwache» Lösungen der Differentialgleichungen. Bei unseren Voraussetzungen über die Koeffizientenfunktionen sind sie auf Grund des Weylschen Lemmas von selbst dann auch »starke» Lösungen.

2) Man kann auch einen rein funktionalanalytischen Beweis anstreben, der die Konvergenzbetrachtungen vermeidet: dabei hat man an Stelle der Greenschen Funktion (mit der im Falle der Randbedingungen $u=0$. 
$v=0$ noch H. Geppert arbeitet) einen Greenschen Operator heranzuziehen. Indessen kann diese Methode naturgemäss nicht die von uns gewonnenen Abschätzungen bzw. Approximationen liefern.

Technische Universität Berlin

Deutschland

\section{Literatur}

[1] Courant, R., und D. Hilbert: Methoden der mathematischen Physik. I. Grundlehren der mathematischen Wissenschaften 12, Verlag von Julius Springer, Berlin, 1924.

[2] -"- -"- Methoden der mathematischen Physik. II. - Grundlehren der mathematischen Wissenschaften 48, Verlag von Julius Springer, Berlin, 1937.

[3] Mонг, E.: Eigenwerte gekoppelter nicht-selbstadjungierter elliptischer Differentialgleichungen zweiter Ordnung. - Math. Nachr. 16. 1957, S. 1-49.

[4] Pólya, G., und G. Szegö: Aufgaben und Lehrsätze aus der Analysis. I. - Grundlehren der mathematischen Wissenschaften 19, Verlag von .Julius Springer, Berlin, 1925 . 